\title{
Mechanism of Dehydration of Phenols on Noble Metals via First-Principles Microkinetic Modeling
}

\section{Supporting Information}

\author{
Geun Ho Gu, ${ }^{[\mathrm{a}]}$ Charles A. Mullen, ${ }^{[\mathrm{b}]}$ Akwasi A. Boateng, ${ }^{[\mathrm{b}]}$ and Dionisios G. Vlachos ${ }^{[\mathrm{a}]}$

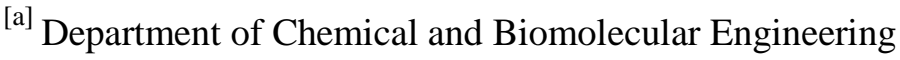 \\ Catalysis Center for Energy Innovation and Center for Catalytic Science and Technology \\ University of Delaware, Newark, DE 19716 \\ E-mail: vlachos@udel.edu, tel.302-831-2830 \\ ${ }^{[b]}$ Sustainable Biofuels and Coproducts \\ Eastern Regional Research Center, ARS, USDA \\ Wyndmoor, PA, 19038 (USA)
}

\section{Section 1: Nomenclature of the $p$-cresol Derivatives}

For convenience, we refer to $p$-cresol as " $\mathrm{PC}(\mathrm{OH})$ ", where $\mathrm{PC}$ stands for the methyl ring without $\mathrm{OH}$. The $p$-cresol derivatives undergoing $\mathrm{O}-\mathrm{H}$ scission are referred to as "PC(O)". Similarly, PC refers to derivatives after $\mathrm{C}-\mathrm{OH}$ scission. Following the $\alpha$-carbon hydrogenation of PC, the molecules are referred to as TOL. We number the ring carbons as shown in Figure S1. Fully hydrogenated molecules are: $\mathrm{MCH}(\mathrm{OH})$, methyl cyclohexanol; $\mathrm{MCH}(\mathrm{O})$, methyl cyclohexanone; and $\mathrm{MCH}$, methyl cyclohexane.<smiles>Cc1ccc(O)cc1</smiles>

Figure S1. Numbering of the ring carbon atoms. Structures from left to right: $\mathrm{PC}(\mathrm{OH}), \mathrm{PC}(\mathrm{O}), \mathrm{PC}$, and TOL. An intermediate $\mathrm{X}$ hydrogenated at ring positions 2, 4, and 6 is denoted as $\mathrm{H} 246 \mathrm{X}$. For example, $\mathrm{H} 23456 \mathrm{PC}(\mathrm{OH})$ refers to $p$-cresol with hydrogenated ring carbon at positions $2,3,4,5$, and 6 . 


\section{Section 2: Generation of the Reaction Network}

We generate the network using the $\mathrm{RING}^{1,2}$ software. The classifications of the elementary reactions are listed in Table S1. The number of associated reactions and intermediates are listed in Table S2. C-C cracking is not considered, as light hydrocarbons are not observed experimentally. ${ }^{3,4}$ The $\mathrm{C}-\mathrm{H}$ and $\mathrm{O}-\mathrm{H}$ reactions are included, as a previous study on $\mathrm{Pt}(111)$ using BEP relations demonstrated low barriers, ${ }^{5}$ and saturated ketone and alcohol were observed experimentally ${ }^{3,4}$. $\mathrm{C}-\mathrm{OH}$ bond scission is a main elementary reaction type for deoxygenation of $p$-cresol.

Table S1. Reaction rules and examples of input into RING.

Reaction rules
ring carbon, ${\mathrm{C}-\mathrm{H}^{\mathrm{a}}}^{\mathrm{O}}$
$\mathrm{O}-\mathrm{H}$ bond scission
previous benzene dehydrogenation study shows it disfavored. ${ }^{6}$ Additionally, the high
hydrogen to $p$-cresol ratio used in the experiment results in high hydrogen coverage,
disfavoring surface intermediates with $\mathrm{H}_{-}$-deficient ring carbons.

Table S2. Number of reactions and intermediates in the HDO reaction network of $p$-cresol on Pt(111). The table on the left lists the intermediates in each functional form similar to Scheme 1.

\begin{tabular}{ccc|cc}
$\begin{array}{c}\text { Functional form } \\
\text { of } \alpha \text {-carbon }\end{array}$ & $\begin{array}{c}\text { Surface } \\
\text { species }\end{array}$ & $\begin{array}{c}\text { Stable gas } \\
\text { species }\end{array}$ & Reaction & Count \\
\hline $\begin{array}{c}\text { Alcohol }(\mathrm{OH}) \\
\text { Ketone }(\mathrm{O})\end{array}$ & 64 & 17 & Adsorption & 36 \\
$\begin{array}{c}\text { H-deficient/ } \\
\text { Dehydroxylated }\end{array}$ & 20 & 5 & C-H scission/formation & 400 \\
$\begin{array}{c}\text { Hydrocarbon/ } \\
\text { Toluene }\end{array}$ & 40 & 10 & O-H scission/formation & 32 \\
Total & 144 & 32 & Total & 500
\end{tabular}




\section{Section 3: Parameterization of the Microkinetic Model}

Figure S2 shows the minimum energy path as the ring is being hydrogenated. At each degree of hydrogenation, there are multiple positions where $\mathrm{H}$ can be added. The energy level of the most stable species is shown in red and subsequent hydrogenation starts from this species. The reaction energies and barriers studied by DFT are summarized in Table S3 and Table S4.

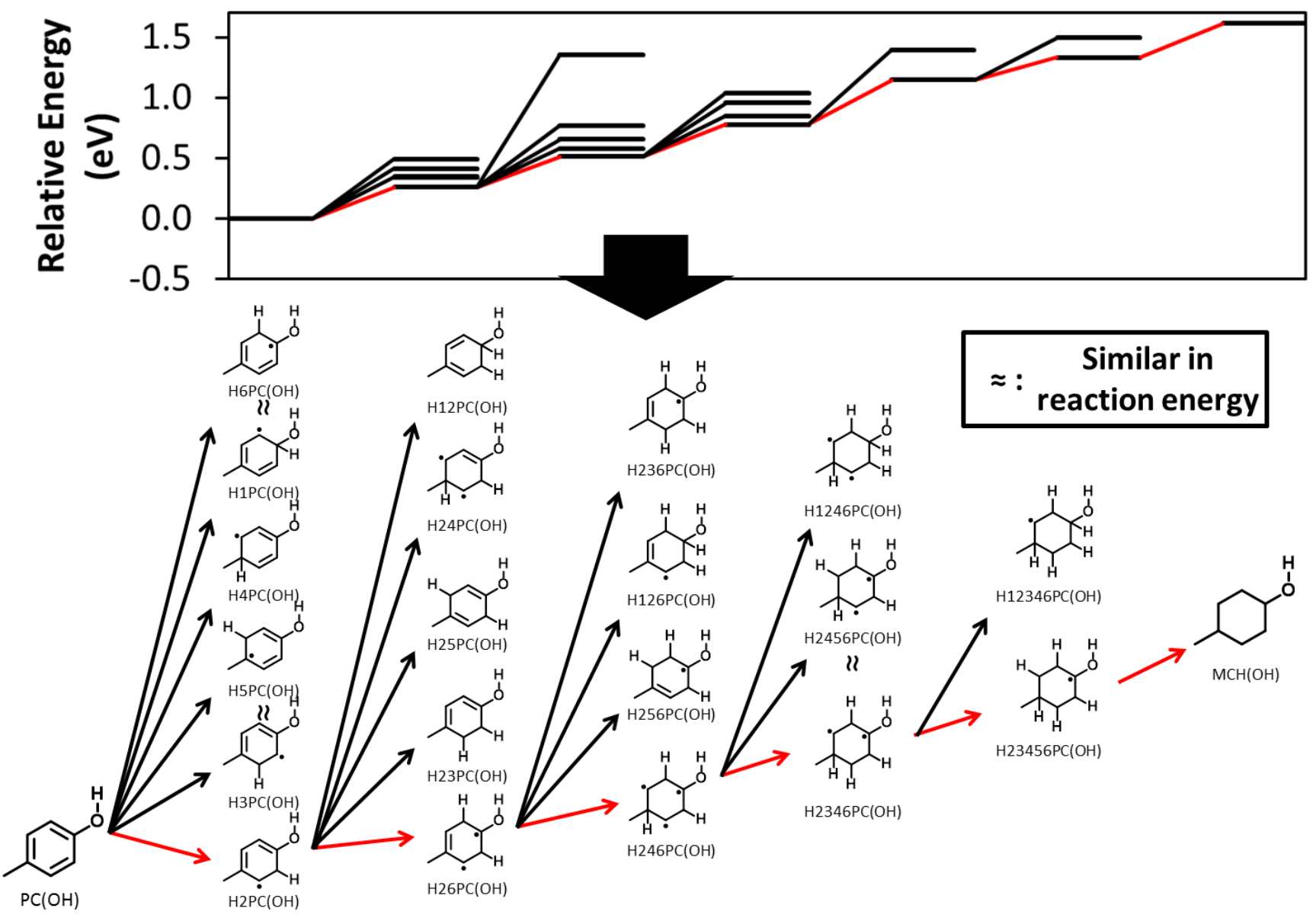

Figure S2. Reaction energy profile (top) of hydrogenation of $p$-cresol $(\mathrm{PC}(\mathrm{OH})$ ) following the minimum energy path (red arrows) and corresponding species (bottom) as hydrogens are being added. 
Table S3. C-H and O-H scission reactions used for development of BEP/TSS relations.

\begin{tabular}{|c|c|c|c|c|}
\hline Type & Reactant & Product & $\begin{array}{l}\text { Reaction } \\
\text { energy }(\mathrm{eV})\end{array}$ & $\begin{array}{l}\text { Barrier } \\
(\mathrm{eV})\end{array}$ \\
\hline \multirow[t]{29}{*}{$\mathrm{C}-\mathrm{H}$} & $\mathrm{H} 2 \mathrm{PC}(\mathrm{OH})(\mathrm{S})$ & $\mathrm{PC}(\mathrm{OH})(\mathrm{S})$ & -0.26 & 0.85 \\
\hline & $\mathrm{H} 26 \mathrm{PC}(\mathrm{OH})(\mathrm{S})$ & $\mathrm{H} 2 \mathrm{PC}(\mathrm{OH})(\mathrm{S})$ & -0.25 & 0.78 \\
\hline & $\mathrm{H} 246 \mathrm{PC}(\mathrm{OH})(\mathrm{S})$ & $\mathrm{H} 26 \mathrm{PC}(\mathrm{OH})(\mathrm{S})$ & -0.27 & 0.63 \\
\hline & $\mathrm{H} 2346 \mathrm{PC}(\mathrm{OH})(\mathrm{S})$ & $\mathrm{H} 246 \mathrm{PC}(\mathrm{OH})(\mathrm{S})$ & -0.37 & 0.57 \\
\hline & $\mathrm{H} 23456 \mathrm{PC}(\mathrm{OH})(\mathrm{S})$ & $\mathrm{H} 2346 \mathrm{PC}(\mathrm{OH})(\mathrm{S})$ & -0.19 & 0.76 \\
\hline & $\mathrm{MCHOH}(\mathrm{S})$ & $\mathrm{H} 23456 \mathrm{PC}(\mathrm{OH})(\mathrm{S})$ & -0.28 & 0.55 \\
\hline & $\mathrm{H} 1 \mathrm{PC}(\mathrm{OH})(\mathrm{S})$ & $\mathrm{PC}(\mathrm{OH})(\mathrm{S})$ & -0.49 & 0.78 \\
\hline & $\mathrm{H} 3 \mathrm{PC}(\mathrm{OH})(\mathrm{S})$ & $\mathrm{PC}(\mathrm{OH})(\mathrm{S})$ & -0.34 & 0.73 \\
\hline & $\mathrm{H} 4 \mathrm{PC}(\mathrm{OH})(\mathrm{S})$ & $\mathrm{PC}(\mathrm{OH})(\mathrm{S})$ & -0.41 & 0.78 \\
\hline & $\mathrm{H} 5 \mathrm{PC}(\mathrm{OH})(\mathrm{S})$ & $\mathrm{PC}(\mathrm{OH})(\mathrm{S})$ & -0.35 & 0.84 \\
\hline & H6PC(OH)(S) & $\mathrm{PC}(\mathrm{OH})(\mathrm{S})$ & -0.49 & 0.63 \\
\hline & $\mathrm{H} 12 \mathrm{PC}(\mathrm{OH})(\mathrm{S})$ & $\mathrm{H} 2 \mathrm{PC}(\mathrm{OH})(\mathrm{S})$ & -1.10 & 0.14 \\
\hline & $\mathrm{H} 23 \mathrm{PC}(\mathrm{OH})(\mathrm{S})$ & $\mathrm{H} 2 \mathrm{PC}(\mathrm{OH})(\mathrm{S})$ & -0.32 & 0.73 \\
\hline & $\mathrm{H} 24 \mathrm{PC}(\mathrm{OH})(\mathrm{S})$ & $\mathrm{H} 2 \mathrm{PC}(\mathrm{OH})(\mathrm{S})$ & -0.51 & 0.64 \\
\hline & $\mathrm{H} 25 \mathrm{PC}(\mathrm{OH})(\mathrm{S})$ & $\mathrm{H} 2 \mathrm{PC}(\mathrm{OH})(\mathrm{S})$ & -0.40 & 0.84 \\
\hline & H23456PC(S) & $\mathrm{H} 2345 \mathrm{PC}(\mathrm{S})$ & -0.24 & 0.63 \\
\hline & H2345TOL(S) & $\mathrm{H} 2345 \mathrm{PC}(\mathrm{S})$ & -0.05 & 1.15 \\
\hline & H23456TOL(S) & $\mathrm{H} 23456 \mathrm{PC}(\mathrm{S})$ & 0.04 & 1.12 \\
\hline & H345TOL(S) & H34TOL(S) & -0.10 & 0.88 \\
\hline & H2345TOL(S) & H234TOL(S) & -0.36 & 0.66 \\
\hline & H2345TOL(S) & H245TOL(S) & -0.35 & 0.57 \\
\hline & H2345TOL(S) & H345TOL(S) & -0.48 & 0.45 \\
\hline & H2345TOL(S) & H235TOL(S) & -0.40 & 0.55 \\
\hline & H12345TOL(S) & H2345TOL(S) & 0.07 & 0.93 \\
\hline & H23456TOL(S) & H2345TOL(S) & -0.15 & 0.52 \\
\hline & H23456TOL(S) & H2356TOL(S) & -0.06 & 0.86 \\
\hline & H23456TOL(S) & H2346TOL(S) & -0.16 & 0.81 \\
\hline & $\mathrm{MCH}(\mathrm{S})$ & H23456TOL(S) & -0.12 & 0.84 \\
\hline & $\mathrm{MCH}(\mathrm{S})$ & H12345TOL(S) & -0.32 & 0.64 \\
\hline \multirow[t]{8}{*}{$\mathrm{O}-\mathrm{H}$} & $\mathrm{PC}(\mathrm{OH})(\mathrm{S})$ & $\mathrm{PC}(\mathrm{O})(\mathrm{S})^{\mathrm{a}}$ & 0.18 & 0.47 \\
\hline & $\mathrm{PC}(\mathrm{OH})(\mathrm{S})$ & $\mathrm{PC}(\mathrm{O})(\mathrm{S})^{\mathrm{b}}$ & 0.26 & 0.46 \\
\hline & $\mathrm{H} 2 \mathrm{PC}(\mathrm{OH})(\mathrm{S})$ & $\mathrm{H} 2 \mathrm{PC}(\mathrm{O})(\mathrm{S})^{\mathrm{a}}$ & 0.60 & 0.86 \\
\hline & $\mathrm{H} 2 \mathrm{PC}(\mathrm{OH})(\mathrm{S})$ & $\mathrm{H} 2 \mathrm{PC}(\mathrm{O})(\mathrm{S})^{\mathrm{b}}$ & 0.48 & 0.85 \\
\hline & $\mathrm{H} 26 \mathrm{PC}(\mathrm{OH})(\mathrm{S})$ & $\mathrm{H} 26 \mathrm{PC}(\mathrm{O})(\mathrm{S})^{\mathrm{a}}$ & 0.51 & 0.79 \\
\hline & $\mathrm{H} 246 \mathrm{PC}(\mathrm{OH})(\mathrm{S})$ & $\mathrm{H} 246 \mathrm{PC}(\mathrm{O})(\mathrm{S})^{\mathrm{a}}$ & 0.25 & 0.76 \\
\hline & $\mathrm{H} 2346 \mathrm{PC}(\mathrm{OH})(\mathrm{S})$ & $\mathrm{H} 2346 \mathrm{PC}(\mathrm{O})(\mathrm{S})^{\mathrm{a}}$ & 0.40 & 0.61 \\
\hline & $\mathrm{H} 23456 \mathrm{PC}(\mathrm{OH})(\mathrm{S})$ & $\mathrm{H} 23456 \mathrm{PC}(\mathrm{O})(\mathrm{S})^{\mathrm{a}}$ & 0.35 & 0.53 \\
\hline
\end{tabular}



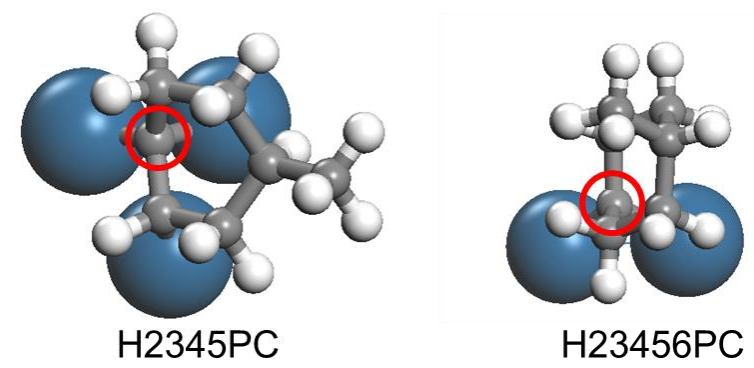

Figure S3. Structures of hydrogenated ring-carbons that lead to H-deficient derivatives with $\mathrm{sp}^{3}$ hybridized $\alpha$-carbon.

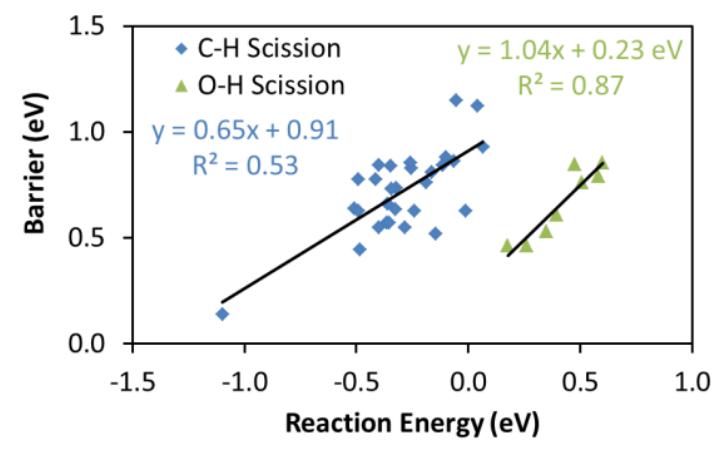

Figure S4. BEP relations for C-H and O-H scission reactions. The training set is presented in Table S3. 
Table S4. C-OH scission reactions used for development of BEP/TSS relations.

$\begin{array}{lllllll}\text { IS } & \text { TS } & \text { FS } & \text { Reactant } & \text { Product } & \begin{array}{l}\text { Reaction } \\ \text { energy }(\mathrm{eV})\end{array} & \text { Barrier }(\mathrm{eV}) \\ 1 & \text { 3-centered } & \mathrm{sp}^{2} \alpha-\mathrm{C} & \mathrm{PC}(\mathrm{OH})(\mathrm{S}) & \mathrm{PC}(\mathrm{S}) & 1.81 & 2.45 \\ 1 & \text { 3-centered } & \mathrm{sp}^{2} \alpha-\mathrm{C} & \mathrm{H} 25 \mathrm{PC}(\mathrm{OH})(\mathrm{S}) & \mathrm{H} 25 \mathrm{PC}(\mathrm{S}) & 1.88 & 2.47 \\ 1 & \text { 3-centered } & \mathrm{sp}^{2} \alpha-\mathrm{C} & \mathrm{H} 34 \mathrm{PC}(\mathrm{OH})(\mathrm{S})-1^{\mathrm{a}} & \mathrm{H} 34 \mathrm{PC}(\mathrm{S})-1 & 1.20 & 1.68 \\ 1 & \text { 3-centered } & \mathrm{sp}^{2} \alpha-\mathrm{C} & \mathrm{H} 34 \mathrm{PC}(\mathrm{OH})(\mathrm{S})-2^{\mathrm{a}} & \mathrm{H} 34 \mathrm{PC}(\mathrm{S})-2 & 1.06 & 1.72 \\ 2 & \text { 3-centered } & \mathrm{sp}^{2} \alpha-\mathrm{C} & \mathrm{H} 2 \mathrm{PC}(\mathrm{OH})(\mathrm{S})-1^{\mathrm{a}} & \mathrm{H} 2 \mathrm{PC}(\mathrm{S}) & 1.66 & 2.15 \\ 2 & \text { 3-centered } & \mathrm{sp}^{2} \alpha-\mathrm{C} & \mathrm{H} 345 \mathrm{PC}(\mathrm{OH})(\mathrm{S}) & \mathrm{H} 345 \mathrm{PC}(\mathrm{S}) & 0.78 & 1.46 \\ 3 & \text { 4-centered } & \mathrm{sp}^{2} \alpha-\mathrm{C} & \mathrm{H} 2 \mathrm{PC}(\mathrm{OH})(\mathrm{S})-2^{\mathrm{a}} & \mathrm{H} 2 \mathrm{PC}(\mathrm{S}) & 1.83^{\mathrm{b}} & 1.75 \\ 3 & \text { 4-centered } & \mathrm{sp}^{2} \alpha-\mathrm{C} & \mathrm{H} 23 \mathrm{PC}(\mathrm{OH})(\mathrm{S}) & \mathrm{H} 23 \mathrm{PC}(\mathrm{S}) & 1.62^{\mathrm{b}} & 1.47 \\ 3 & \text { 4-centered } & \mathrm{sp}^{2} \alpha-\mathrm{C} & \mathrm{H} 26 \mathrm{PC}(\mathrm{OH})(\mathrm{S}) & \mathrm{H} 26 \mathrm{PC}(\mathrm{S}) & 1.71^{\mathrm{b}} & 1.71 \\ 3 & \text { 4-centered } & \mathrm{sp}^{2} \alpha-\mathrm{C} & \mathrm{H} 246 \mathrm{PC}(\mathrm{OH})(\mathrm{S}) & \mathrm{H} 246 \mathrm{PC}(\mathrm{S}) & 1.53^{\mathrm{b}} & 1.55 \\ 3 & \text { 4-centered } & \mathrm{sp}^{2} \alpha-\mathrm{C} & \mathrm{H} 2346 \mathrm{PC}(\mathrm{OH})(\mathrm{S}) & \mathrm{H} 2346 \mathrm{PC}(\mathrm{S}) & 1.34^{\mathrm{b}} & 1.26 \\ 3 & \text { 4-centered } & \mathrm{sp}^{2} \alpha-\mathrm{C} & \mathrm{H} 23456 \mathrm{PC}(\mathrm{OH})(\mathrm{S}) & \mathrm{H} 23456 \mathrm{PC}(\mathrm{S}) & 1.11^{\mathrm{b}} & 0.96 \\ 3 & \text { 4-centered } & \mathrm{sp}^{3} \alpha-\mathrm{C} & \mathrm{H} 2345 \mathrm{PC}(\mathrm{OH})(\mathrm{S}) & \mathrm{H} 2345 \mathrm{PC}(\mathrm{S}) & 0.91 & 1.10 \\ 3 & \text { 4-centered } & \mathrm{sp}^{3} \alpha-\mathrm{C} & \mathrm{H} 3456 \mathrm{PC}(\mathrm{OH})(\mathrm{S}) & \mathrm{H} 2345 \mathrm{PC}(\mathrm{S}) & 0.87 & 1.16 \\ 3 & \text { 4-centered } & \mathrm{sp}^{3} \alpha-\mathrm{C} & \mathrm{H} 23456 \mathrm{PC}(\mathrm{OH})(\mathrm{S}) & \mathrm{H} 23456 \mathrm{PC}(\mathrm{S}) & 0.79 & 0.96 \\ { }^{\mathrm{a}} \text { Same intermediates but adsorbed in different conformation. }{ }^{\mathrm{b}} \operatorname{Thse} \text { reaction energies are higher than the corresponding } \\ \text { barriers at infinite separation but lower at co-adsorbed state, implying an attractive interaction between cresol derivatives } \\ \text { and OH. For C-OH scission reaction energy in Figure 2 is computed at co-adsorbed state. }\end{array}$




\subsection{Vibrational Frequency Calculations and Statistical Mechanical Approximation}

We compute vibrational frequencies of 26 species along the minimum energy hydrogenation path, along with those of $\mathrm{H}_{2} \mathrm{O}, \mathrm{OH}$, and $\mathrm{H}$. The frequencies of 26 additional species are calculated for MKM refinement. Vibrational frequencies and binding energies are presented in Table S5.

Table S5. Binding energies and vibrational frequencies of species computed via DFT.

\begin{tabular}{|c|c|c|}
\hline Species & $\begin{array}{l}\text { B.E. } \\
(\mathrm{eV})\end{array}$ & $v\left(\mathrm{~cm}^{-1}\right)$ \\
\hline $\mathrm{PC}(\mathrm{S})$ & 3.44 & $\begin{array}{l}3079,3073,3059,3057,3053,3023,2937,1436,1429,1405,1359,1346,1300,1273,1240,1177, \\
1136,1064,1024,1004,931,926,902,882,871,826,796,719,626,568,542,522,480,417,372, \\
328,298,226,210,185,154,111\end{array}$ \\
\hline $\mathrm{H} 2 \mathrm{PC}(\mathrm{S})$ & 4.68 & $\begin{array}{l}3077,3043,3037,3014,3010,3002,2935,2920,1421,1420,1415,1373,1350,1293,1273,1215, \\
1170,1136,1131,1082,1042,1012,996,948,935,926,899,859,834,778,692,598,576,517, \\
493,485,416,348,335,328,241,203,192,140,119\end{array}$ \\
\hline $\mathrm{H} 26 \mathrm{PC}(\mathrm{S})$ & 5.90 & $\begin{array}{l}3067,3050,3020,2987,2985,2951,2927,2912,2894,1428,1420,1413,1405,1383,1348,1299, \\
1266,1206,1198,1168,1130,1109,1052,1038,1000,994,958,945,919,899,870,831,778,680 \text {, } \\
593,579,506,483,405,375,343,321,313,251,198,152,130,94\end{array}$ \\
\hline H234PC(S) & 4.77 & $\begin{array}{l}3056,3044,3036,3009,2979,2963,2958,2957,2955,2821,1452,1447,1428,1404,1358,1314 \text {, } \\
1310,1273,1251,1230,1204,1166,1123,1110,1085,1077,1016,999,961,955,930,897,884, \\
868,831,752,696,608,594,527,496,433,404,303,265,223,206,191,114,74,59\end{array}$ \\
\hline $\mathrm{H} 235 \mathrm{PC}(\mathrm{S})$ & 5.05 & $\begin{array}{l}3064,3031,2988,2986,2971,2970,2950,2915,2908,2875,1434,1423,1416,1398,1385,1341, \\
1302,1278,1225,1204,1182,1164,1146,1119,1096,1074,1029,1007,970,967,930,901,878, \\
850,823,759,675,583,506,476,445,429,343,318,276,270,237,219,157,99,63\end{array}$ \\
\hline H236PC(S) & 4.85 & $\begin{array}{l}3029,3026,2999,2987,2969,2947,2933,2908,2899,2868,1429,1417,1408,1400,1393,1348, \\
1302,1273,1240,1202,1184,1168,1160,1095,1078,1065,1026,1000,965,946,932,912,892, \\
853,798,756,676,576,558,486,448,402,362,347,304,296,248,219,147,114,96\end{array}$ \\
\hline H245PC(S) & 5.16 & $\begin{array}{l}3052,3030,3026,3010,3003,2985,2952,2919,2911,2785,1447,1442,1422,1402,1355,1310, \\
1277,1269,1248,1239,1196,1151,1146,1121,1085,1042,1031,1022,990,987,927,903,873, \\
870,811,768,684,591,579,502,483,393,379,320,269,245,213,159,133,85,58\end{array}$ \\
\hline H246PC(S) & 7.72 & $\begin{array}{l}3054,3044,3001,2997,2980,2968,2966,2915,2892,2814,1449,1447,1425,1408,1351,1293, \\
1283,1266,1234,1188,1182,1170,1105,1095,1079,1025,1015,998,991,982,924,915,882, \\
851,782,762,726,612,576,574,558,418,403,361,317,260,209,194,164,117,108\end{array}$ \\
\hline $\mathrm{H} 2345 \mathrm{PC}(\mathrm{S})$ & 2.16 & $\begin{array}{l}3041,3030,3014,2993,2966,2959,2954,2948,2935,2907,2791,1449,1447,1436,1407,1394, \\
1362,1324,1310,1274,1267,1239,1232,1211,1183,1150,1123,1096,1092,1059,1015,987, \\
959,909,901,869,845,831,763,688,525,512,481,377,357,327,248,232,216,153,128,95 \\
69,39\end{array}$ \\
\hline $\mathrm{H} 2346 \mathrm{PC}(\mathrm{S})$ & 5.96 & $\begin{array}{l}3052,3043,3012,3010,2982,2966,2957,2938,2905,2864,2728,1450,1447,1431,1407,1391, \\
1358,1314,1298,1270,1247,1212,1199,1184,1164,1126,1101,1091,1028,1018,1006,985, \\
944,925,903,888,829,792,742,685,602,574,537,419,403,379,302,264,217,185,158,113, \\
78,65\end{array}$ \\
\hline $\mathrm{H} 2356 \mathrm{PC}(\mathrm{S})$ & 6.07 & $\begin{array}{l}3045,3030,3009,3006,2986,2971,2950,2932,2928,2921,2916,1438,1430,1421,1412,1404 \\
1390,1345,1316,1288,1236,1225,1208,1187,1181,1154,1124,1099,1060,1048,998,973, \\
960,935,884,865,839,794,754,692,530,485,440,431,343,305,286,269,261,233,181,92 \\
60,51\end{array}$ \\
\hline $\mathrm{H} 23456 \mathrm{PC}(\mathrm{S})$ & 4.75 & $\begin{array}{l}3046,3038,3003,3001,2969,2962,2960,2949,2934,2928,2715,2708,1456,1453,1431,1422, \\
1394,1372,1366,1325,1319,1312,1281,1260,1249,1205,1184,1152,1138,1093,1081,1046, \\
1036,975,969,936,931,916,864,850,757,753,644,567,477,439,385,379,329,323,218,171, \\
156,146,105,54,45\end{array}$ \\
\hline $\mathrm{PC}(\mathrm{O})(\mathrm{S})$ & 3.26 & $\begin{array}{l}3068,3062,3049,3043,3016,3001,2929,1655,1425,1420,1402,1353,1332,1288,1267,1242, \\
1152,1138,1069,1034,1011,987,956,935,897,882,839,733,724,681,582,574,519,467,427, \\
408,404,324,310,239,191,138,117,104,94\end{array}$ \\
\hline $\mathrm{H} 2 \mathrm{PC}(\mathrm{O})(\mathrm{S})$ & 2.78 & $\begin{array}{l}3070,3040,3018,3013,3007,2989,2920,2887,1513,1425,1413,1403,1382,1350,1313,1277, \\
1273,1229,1156,1128,1112,1052,1012,1004,975,935,914,895,861,784,704,695,591,560, \\
546,445,429,422,376,325,312,255,253,208,124,119,91,55\end{array}$ \\
\hline $\mathrm{H} 26 \mathrm{PC}(\mathrm{O})(\mathrm{S})$ & 3.16 & $\begin{array}{l}3052,3046,3020,2996,2984,2932,2918,2896,2891,1639,1433,1415,1406,1375,1361,1348, \\
1328,1295,1274,1227,1175,1161,1134,1093,1045,1022,1002,975,949,923,893,832,747, \\
718,696,575,544,467,450,425,371,333,308,262,233,219,192,123,92,76,69\end{array}$ \\
\hline
\end{tabular}




\begin{abstract}
$\mathrm{H} 246 \mathrm{PC}(\mathrm{O})(\mathrm{S}) \quad 4.65$
$\mathrm{H} 2346 \mathrm{PC}(\mathrm{O})(\mathrm{S}) \quad 2.83$

$\mathrm{MCH}(\mathrm{O})(\mathrm{S})$

3057, 3050, 2995, 2987, 2977, 2970, 2968, 2905, 2899, 2759, 1652, 1448, 1444, 1375, 1362, 1346, $1311,1299,1284,1269,1251,1199,1167,1155,1109,1071,1060,1038,1004,1002,971,964$, $927,866,811,776,753,699,588,546,511,509,437,405,322,288,250,241,207,174,111,109$, 63,62

3049, 3043, 3025, 3004, 2966, 2962, 2940, 2927, 2885, 2883, 2637, 1659, 1449, 1448, 1394, 1371, 1364, 1352, 1321, 1317, 1292, 1273, 1258, 1223, 1206, 1179, 1135, 1128, 1085, 1058, 1038, 1022, $979,974,921,901,866,780,769,714,680,569,518,485,447,403,379,279,246,214,203,151$, $114,90,67,51,29$

$.353056,3050,3008,3005,2983,2978,2972,2957,2937,2930,2660,2650,1645,1455,1450,1390$, $1375,1373,1370,1356,1329,1323,1313,1299,1267,1244,1229,1203,1191,1150,1108,1081$, $1061,1044,992,985,932,904,882,872,770,719,682,664,504,478,432,405,374,344,221$, $216,172,109,96,91,58,38,25,18$
\end{abstract}

$\mathrm{PC}(\mathrm{OH})(\mathrm{S}) \quad 2.44$

$\mathrm{H} 2 \mathrm{PC}(\mathrm{OH})(\mathrm{S})$

$\mathrm{H} 26 \mathrm{PC}(\mathrm{OH})(\mathrm{S}) \quad 5.00$

$\mathrm{H} 234 \mathrm{PC}(\mathrm{OH})(\mathrm{S}) \quad 3.16$

$\mathrm{H} 235 \mathrm{PC}(\mathrm{OH})(\mathrm{S})$

3.74

$\mathrm{H} 236 \mathrm{PC}(\mathrm{OH})(\mathrm{S})$

$\mathrm{H} 245 \mathrm{PC}(\mathrm{OH})(\mathrm{S}) \quad 3.86$

$\mathrm{H} 246 \mathrm{PC}(\mathrm{OH})(\mathrm{S}) \quad 6.63$

$\mathrm{H} 256 \mathrm{PC}(\mathrm{OH})(\mathrm{S}) \quad 3.72$

$\mathrm{H} 346 \mathrm{PC}(\mathrm{OH})(\mathrm{S}) \quad 3.88$

$\mathrm{H} 356 \mathrm{PC}(\mathrm{OH})(\mathrm{S}) \quad 3.81$

$\mathrm{H} 456 \mathrm{PC}(\mathrm{OH})(\mathrm{S}) \quad 3.23$

$443410,3095,3082,3053,3043,3036,3007,2933,1452,1425,1421,1401,1364,1353,1326,1283$, $1262,1201,1151,1136,1079,1038,1001,951,939,922,909,868,842,741,703,632,580,548$, $507,445,427,412,392,367,319,310,238,203,188,137,124,102$

$3300,3085,3048,3028,3024,3022$, 3000, 2928, 2889, 1423, 1417, 1409, 1401, 1349, 1335, 1305 , 1299, 1267, 1235, 1172, 1139, 1102, 1085, 1051, 1010, 1009, 981, 937, 921, 918, 876, 805, 730, $690,610,579,558,493,454,437,421,415,344,319,294,247,242,203,132,121,114$

$3479,3062,3050,3033,2988,2987,2917,2916,2877,2871,1431,1418,1411,1406,1389,1350$, $1345,1312,1300,1271,1235,1182,1145,1129,1092,1063,1038,1011,1008,981,960,937,896$ $865,842,748,681,594,561,510,473,441,423,407,346,319,314,277,259,238,196,109,99$,

$3308,3050,3043,3007,3004,2992,2965,2959,2932,2931,2666,1452,1445,1414,1385,1357$, $1335,1330,1314,1301,1277,1264,1248,1183,1157,1137,1117,1087,1054,1048,1011,987$, $981,914,900,880,850,771,716,676,608,536,505,469,460,426,412,392,268,247,236,223$, $198,140,99,81,70$

3285, 3033, 3029, 3009, 2978, 2975, 2945, 2926, 2909, 2862, 2841, 1424, 1420, 1416, 1398, 1396, 1340, 1328, 1311, 1303, 1285, 1240, 1218, 1190, 1176, 1129, 1113, 1092, 1084, 1025, 1021, 990, $970,952,906,870,826,757,732,663,575,541,514,463,425,407,405,327,310,283,249,245$, $220,191,108,101,76$

3495, 3022, 3020, 3001, 2972, 2965, 2960, 2913, 2894, 2890, 2873, 1427, 1414, 1406, 1400, 1395 , $1350,1341,1314,1301,1289,1243,1227,1182,1167,1127,1110,1082,1078,1022,1019,1004$, $974,957,885,877,837,761,733,657,568,543,483,471,416,408,403,333,310,297,253,236$, $231,189,108,104,70$

$3525,3054,3032,3015,3006,2978,2975,2955,2896,2888,2744,1448,1443,1419,1399,1357$, $1338,1312,1286,1278,1262,1239,1215,1173,1143,1134,1105,1062,1057,1041,1017,1007$, $981,977,936,890,860,790,753,700,616,583,557,456,438,431,388,368,303,265,246,230$, $216,133,108,91,68$

3508, 3056, 3052, 3011, 2992, 2971, 2941, 2901, 2890, 2857, 2783, 1447, 1443, 1402, 1386, 1351, $1335,1312,1289,1280,1276,1266,1189,1172,1127,1114,1093,1073,1037,1036,1001,998$, 973, 966, 941, 871, 837, 834, 775, 708, 606, 583, 511, 506, 458, 422, 390, 369, 332, 302, 251, 231, $206,168,113,104,88$

3506, 3025, 3013, 2999, 2987, 2971, 2947, 2902, 2870, 2855, 2808, 1426, 1410, 1401, 1393, 1391, $1345,1337,1322,1288,1279,1245,1222,1183,1167,1143,1092,1080,1058,1022,1018,998$, $974,955,882,880,843,762,736,653,569,543,494,458,416,410,399,333,309,299,249,240$, $227,210,107,101,79$

$.883598,3050,3035,3018,3005,3001,2959,2957,2898,2888,2750,1446,1443,1420,1397,1356$, $1331,1307,1284,1275,1267,1245,1214,1182,1145,1131,1098,1081,1046,1042,1010,1005$, $981,974,939,892,861,789,755,698,618,587,559,453,440,408,382,364,305,259,244,234$, $218,134,106,92,62$

3486, 3019, 3016, 3014, 2972, 2965, 2959, 2921, 2902, 2844, 2781, 1422, 1416, 1414, 1396, 1388, 1342, 1332, 1307, 1295, 1265, 1250, 1207, 1185, 1170, 1123, 1107, 1097, 1051, 1025, 1020, 994 , $972,944,913,870,829,757,737,663,579,544,470,454,422,407,396,330,307,298,252,244$, $228,217,110,103,78$

3550, 3052, 3046, 3008, 2999, 2997, 2979, 2964, 2953, 2934, 2702, 1452, 1444, 1414, 1387, 1358, 1349, 1319, 1318, 1297, 1277, 1262, 1234, 1174, 1156, 1132, 1089, 1061, 1048, 1040, 1010, 983 , $974,916,894,880,855,775,722,677,606,553,496,473,452,436,413,387,276,252,234,214$, $199,151,98,85,68$ 
$\mathrm{H} 2345 \mathrm{PC}(\mathrm{OH})(\mathrm{S}) \quad 2.16$

$\mathrm{H} 2356 \mathrm{PC}(\mathrm{OH})(\mathrm{S}) \quad 4.56$

$\mathrm{H} 2456 \mathrm{PC}(\mathrm{OH})(\mathrm{S}) \quad 4.68$

$\mathrm{H} 3456 \mathrm{PC}(\mathrm{OH})(\mathrm{S}) \quad 1.93$

$\mathrm{H} 23456 \mathrm{PC}(\mathrm{OH})(\mathrm{S}) \quad 2.92$

$\mathrm{MCH}(\mathrm{OH})(\mathrm{S})$

TOL(S)

H2TOL(S)

H26TOL(S)

H34TOL(S)

H234TOL(S)

H235TOL(S)

H245TOL(S)

H246TOL(S)

H345TOL(S)

4.69 $1381,1358,1335,1322,1301,1286,1280,1273,1230,1195,1148,1130,1118,1099,1071,1048$, $1037,1012,981,969,929,899,875,822,762,746,660,596,500,485,461,455,414,389,354$, $269,247,239,225,200,143,88,81,72$

3506, 3024, 3002, 2988, 2984, 2960, 2949, 2932, 2915, 2909, 2905, 2898, 1434, 1425, 1424, 1415, $1405,1400,1346,1340,1321,1298,1294,1256,1241,1228,1211,1178,1136,1103,1091,1070$, $1027,1018,999,971,893,871,866,817,734,730,646,519,489,463,452,409,404,320,282$, $260,252,237,228,222,159,79,71,29$

$3520,3051,3045,2996,2994,2970,2962,2921,2894,2894,2829,2607,1447,1445,1409,1387$, $1376,1354,1337,1316,1304,1285,1277,1264,1232,1184,1143,1135,1123,1069,1066,1043$, $1031,1014,979,969,929,896,873,829,762,739,662,595,500,486,460,432,415,385,352$, $268,251,238,220,194,139,94,73,67$

3615, 3046, 3029, 3012, 2996, 2992, 2984, 2950, 2931, 2895, 2876, 2686, 1451, 1447, 1418, 1403, $1391,1361,1338,1320,1310,1297,1283,1278,1251,1215,1178,1153,1144,1098,1077,1069$, $1042,1013,991,982,935,908,872,828,741,731,655,581,528,458,426,390,375,344,318$, $257,238,231,193,152,96,73,57,31$

3401, 3049, 3046, 3008, 3005, 2966, 2963, 2962, 2954, 2896, 2893, 2625, 2614, 1455, 1450, 1405, $1391,1372,1364,1358,1339,1326,1313,1305,1290,1281,1259,1229,1189,1150,1142,1090$, 1076, 1070, 1050, 1046, 987, 976, 921, 917, 910, 874, 770, 741, 726, 632, 512, 478, 461, 458, 393, $374,372,310,278,241,213,161,149,97,75,60,48$

$1.313635,3048,3042,3015,3012,3008,2991,2964,2949,2944,2943,2598,2581,2124,1456,1451$, $1427,1410,1373,1371,1354,1351,1330,1318,1309,1295,1286,1253,1236,1198,1180,1150$, 1139, 1130, 1099, 1070, 1057, 1046, 1037, 976, 972, 943, 930, 900, 866, 796, 757, 748, 636, 498, $462,444,389,372,340,323,259,224,211,135,102,100,78,55,39,29$

$2.33 \quad 3098,3086,3085,3053,3045,3039,3019,2941,1436,1423,1415,1387,1368,1352,1325,1307$, $1261,1144,1140,1087,1015,1004,966,928,924,909,891,866,837,829,721,631,566,529$, $503,474,394,370,338,333,231,193,161,140,118$

$3.663073,3039,3030,3027,3010,3008,2994,2923,2864,1426,1417,1407,1405,1349,1327,1320$, $1306,1273,1210,1155,1138,1089,1051,1028,1000,992,972,946,925,892,872,819,795,712$ $613,562,546,491,444,411,346,333,315,249,202,156,132,112$

$4.913063,3046,3028,3021,2979,2958,2916,2904,2856,2853,1432,1415,1408,1403,1385,1349$, $1331,1312,1307,1277,1204,1178,1142,1118,1059,1052,1040,995,993,976,952,937,875$, $865,834,800,717,592,552,519,452,430,357,328,322,299,266,200,125,122,91$

$2.81 \quad 3068,3061,3049,3044,3026,3018,3005,2965,2901,2565,1446,1444,1415,1399,1366,1349$, $1321,1289,1268,1245,1235,1196,1148,1108,1080,1071,1019,1009,990,962,919,900,886$, $844,826,799,734,605,558,536,481,443,376,332,260,231,205,145,131,86,71$

$3058,3046,3039,3023,3015,3005,3000,2960,2913,2885,2624,1450,1448,1419,1405,1381$, $1364,1350,1330,1308,1296,1263,1259,1199,1155,1139,1097,1090,1045,1023,991,980$, $956,906,891,879,852,823,754,682,584,527,511,453,409,361,265,248,216,189,124,97$, 73,68

$3.693024,3017,3009,2987,2972,2970,2934,2905,2900,2875,2855,1423,1417,1417,1396,1391$, $1340,1323,1315,1293,1282,1233,1207,1181,1172,1115,1105,1094,1055,1018,988,966$, $959,946,884,859,828,814,741,683,554,531,463,454,405,342,322,280,246,242,206,130$, 107,80

$3.813051,3033,3011,3005,3001,2987,2955,2953,2891,2865,2781,1447,1444,1416,1397,1355$, 1323, 1307, 1288, 1277, 1270, 1243, 1214, 1173, 1146, 1114, 1075, 1061, 1037, 1026, 1008, 986, $973,960,915,866,851,822,771,736,597,561,534,479,431,373,328,271,250,217,141,137$, 93,68

$6.53 \quad 3050,3045,3008,3001,2999,2962,2909,2908,2855,2853,2746,1446,1443,1397,1382,1350$, $1321,1321,1287,1286,1272,1266,1177,1169,1114,1094,1091,1039,1031,1025,1001,994$, $979,953,930,845,840,828,787,766,601,586,548,493,467,363,335,306,259,221,185,140$, 111,99

$3.193051,3031,3004,3001,2994,2988,2955,2950,2890,2886,2596,1448,1445,1413,1399,1364$, $1348,1318,1305,1281,1266,1232,1224,1220,1140,1129,1110,1089,1062,1011,1009,1000$, $969,937,874,870,844,812,777,702,563,535,463,460,417,389,306,240,236,199,149,132$, 80,63 


\begin{tabular}{|c|c|c|}
\hline H2345TOL(S) & 1.84 & $\begin{array}{l}3048,3028,3007,3002,2989,2985,2959,2949,2912,2899,2891,2739,1451,1446,1414,1401, \\
1397,1361,1335,1327,1305,1303,1289,1281,1249,1216,1173,1156,1137,1090,1077,1057, \\
\text { 1021, 992, 974, 961, 924, 903, 857, 823, 809, 732, 657, 549, 496, 473, 411, 369, 337, 268, 244, 189, } \\
143,118,74,60,22\end{array}$ \\
\hline H2346TOL(S) & 4.60 & $\begin{array}{l}3044,3038,3001,2999,2989,2979,2959,2910,2909,2877,2790,2581,1450,1447,1406,1383, \\
1374,1355,1333,1322,1295,1287,1281,1265,1224,1179,1137,1126,1097,1091,1044,1037 \\
1027,1007,975,956,930,891,844,837,797,756,676,595,534,474,463,424,359,274,261, \\
232,200,153,111,81,70\end{array}$ \\
\hline H2356TOL(S) & 4.50 & $\begin{array}{l}3021,2990,2989,2982,2980,2964,2946,2921,2917,2912,2901,2898,1430,1425,1421,1409, \\
1402,1395,1345,1324,1311,1305,1292,1235,1227,1218,1202,1170,1115,1098,1092,1078, \\
1026,992,976,968,866,864,857,818,767,722,698,503,480,448,432,351,280,259,251,245 \text {, } \\
227,161,82,76,24\end{array}$ \\
\hline H12345TOL(S) & 3.01 & $\begin{array}{l}3042,3025,3012,2994,2968,2946,2922,2921,2915,2880,2877,2600,2453,1442,1440,1424, \\
1392,1383,1367,1360,1336,1312,1305,1291,1260,1251,1228,1202,1193,1172,1108,1105, \\
1086,1084,1048,1025,1010,962,945,906,870,834,817,809,746,618,471,462,440,408,318, \\
310,252,221,215,141,117,82,62,39\end{array}$ \\
\hline H23456TOL(S) & 2.88 & $\begin{array}{l}3047,3042,3003,2999,2973,2965,2949,2929,2925,2889,2885,2604,2586,1455,1449,1399, \\
1385,1368,1360,1356,1335,1333,1315,1296,1292,1281,1243,1226,1184,1144,1105,1098 \\
\text { 1097, 1053, 1042, 1039, 972, 963, 936, 913, 889, 847, 824, 754, 731, 639, 547, 449, 445, 387, 380, } \\
\text { 305, 294, 215, 164, 153, 102, 86, 65, } 49\end{array}$ \\
\hline $\mathrm{MCH}(\mathrm{S})$ & 1.12 & $\begin{array}{l}3048,3047,3013,3008,3006,2995,2990,2966,2945,2944,2931,2561,2535,2471,1456,1449, \\
1424,1411,1365,1354,1350,1340,1337,1330,1304,1302,1296,1292,1256,1236,1186,1170, \\
1145,1112,1110,1066,1050,1028,1014,962,945,936,878,850,833,789,747,745,591,468, \\
447,390,375,304,222,208,184,113,98,80,47,40,29\end{array}$ \\
\hline $\mathrm{H} 2 \mathrm{O}(\mathrm{S})$ & 0.46 & $3690,3583,1546,525,507,160,92,92,54$ \\
\hline $\mathrm{OH}(\mathrm{S})$ & 2.99 & $3638,924,510,119,96,52$ \\
\hline $\mathrm{H}(\mathrm{S})$ & 0.54 & $1097,627,597$ \\
\hline
\end{tabular}

A statistical mechanical approximation $\operatorname{method~}^{7-9}$ is used to estimate the thermodynamics of surface species. The enthalpy of each surface species $\left(\Delta H_{i, s u r f}\right)$ is computed by subtracting the DFT binding energy from the enthalpy of formation of the corresponding gas-phase species as shown below:

$$
\Delta H_{i, s u r f}=\Delta H_{i, g a s, G 4}-Q(T=0)_{i, V A S P}-Q_{i}(T)
$$

Here, $Q_{i}(T=0)_{i, V A S P}$ is the $0 \mathrm{~K}$ binding energy (without zero-point energy correction) obtained from periodic DFT calculations using the VASP software, and $Q_{i}(T)$ is a temperature dependent correction approximated using statistical mechanics to account for the change in degrees of freedom. $0 \mathrm{~K}$ binding energies are listed in Table S6. For the chemisorbed $p$-cresol derivatives, $Q_{i}(T)$ is given by the loss of 3 translational $(3 \times 0.5 R T)$ and rotational $(3 \times 0.5 R T)$ degrees of freedom, and a gain of 6 vibrational degrees of freedom $(6 \times R T)$ :

$$
Q_{i}(T)=-1.5 R T-1.5 R T+6 R T=3 R T
$$

The entropy of each surface species is calculated from the following approximation reviewed in Salciccioli et al. ${ }^{9}$

$$
\begin{gathered}
\Delta S_{i, \text { surf }}=F_{\text {loc }}\left(\Delta S_{i, g a s, G 4}-S_{i, \text { trans }, 3 D}\right) \\
S_{i, \text { trans }, 3 D}=R\left[\ln \left(\frac{\left(2 \pi m k_{B} T\right)^{3 / 2}}{h^{3}}\right)+\ln \left(\frac{k_{B} T}{P}\right)+\frac{5}{2}\right]
\end{gathered}
$$

Using the above equation, the entropy of surface species, $i,\left(\Delta S_{i, \text { surf }}\right)$ is computed by subtracting the translational entropy $\left(\Delta S_{i, t r a n s, 3 D}\right)$ from the gas-phase entropy $\left(\Delta S_{i, g a s, G 4}\right)$. The multiplying factor $F_{l o c}$ is fitted using known values (here is taken as 0.99). 
Table S6. Binding energies of surface species whose thermodynamics is approximated using the equations above.

\begin{tabular}{|c|c|c|c|c|c|}
\hline Species & B.E. $(\mathrm{eV})$ & Species & B.E. (eV) & Species & B.E. $(\mathrm{eV})$ \\
\hline H3PC(S) & 5.04 & $\mathrm{H} 16 \mathrm{PC}(\mathrm{OH})(\mathrm{S})$ & 2.89 & $\mathrm{H} 12345 \mathrm{PC}(\mathrm{OH})(\mathrm{S})$ & 3.06 \\
\hline $\mathrm{H} 4 \mathrm{PC}(\mathrm{S})$ & 4.57 & $\mathrm{H} 23 \mathrm{PC}(\mathrm{OH})(\mathrm{S})$ & 2.79 & $\mathrm{H} 12346 \mathrm{PC}(\mathrm{OH})(\mathrm{S})$ & 3.06 \\
\hline $\mathrm{H} 23 \mathrm{PC}(\mathrm{S})$ & 4.21 & $\mathrm{H} 24 \mathrm{PC}(\mathrm{OH})(\mathrm{S})$ & 4.99 & $\mathrm{H} 12356 \mathrm{PC}(\mathrm{OH})(\mathrm{S})$ & 2.72 \\
\hline $\mathrm{H} 24 \mathrm{PC}(\mathrm{S})$ & 4.22 & $\mathrm{H} 25 \mathrm{PC}(\mathrm{OH})(\mathrm{S})$ & 2.78 & $\mathrm{H} 12456 \mathrm{PC}(\mathrm{OH})(\mathrm{S})$ & 3.00 \\
\hline $\mathrm{H} 25 \mathrm{PC}(\mathrm{S})$ & 3.80 & $\mathrm{H} 34 \mathrm{PC}(\mathrm{OH})(\mathrm{S})$ & 2.96 & $\mathrm{H} 13456 \mathrm{PC}(\mathrm{OH})(\mathrm{S})$ & 3.07 \\
\hline H34PC(S) & 4.46 & $\mathrm{H} 35 \mathrm{PC}(\mathrm{OH})(\mathrm{S})$ & 5.04 & H1TOL(S) & 3.64 \\
\hline H35PC(S) & 6.52 & $\mathrm{H} 36 \mathrm{PC}(\mathrm{OH})(\mathrm{S})$ & 2.83 & H3TOL(S) & 3.58 \\
\hline H345PC(S) & 4.50 & $\mathrm{H} 45 \mathrm{PC}(\mathrm{OH})(\mathrm{S})$ & 2.86 & H4TOL(S) & 3.61 \\
\hline $\mathrm{H} 3 \mathrm{PC}(\mathrm{O})(\mathrm{S})$ & 4.51 & $\mathrm{H} 46 \mathrm{PC}(\mathrm{OH})(\mathrm{S})$ & 4.98 & H12TOL(S) & 2.84 \\
\hline $\mathrm{H} 4 \mathrm{PC}(\mathrm{O})(\mathrm{S})$ & 2.66 & $\mathrm{H} 56 \mathrm{PC}(\mathrm{OH})(\mathrm{S})$ & 2.84 & H13TOL(S) & 4.93 \\
\hline $\mathrm{H} 23 \mathrm{PC}(\mathrm{O})(\mathrm{S})$ & 2.81 & $\mathrm{H} 123 \mathrm{PC}(\mathrm{OH})(\mathrm{S})$ & 3.08 & H14TOL(S) & 2.65 \\
\hline $\mathrm{H} 24 \mathrm{PC}(\mathrm{O})(\mathrm{S})$ & 3.71 & $\mathrm{H} 124 \mathrm{PC}(\mathrm{OH})(\mathrm{S})$ & 3.75 & H23TOL(S) & 2.75 \\
\hline $\mathrm{H} 25 \mathrm{PC}(\mathrm{O})(\mathrm{S})$ & 3.49 & $\mathrm{H} 125 \mathrm{PC}(\mathrm{OH})(\mathrm{S})$ & 3.97 & H24TOL(S) & 4.93 \\
\hline $\mathrm{H} 34 \mathrm{PC}(\mathrm{O})(\mathrm{S})$ & 3.44 & $\mathrm{H} 126 \mathrm{PC}(\mathrm{OH})(\mathrm{S})$ & 3.29 & H25TOL(S) & 2.76 \\
\hline $\mathrm{H} 35 \mathrm{PC}(\mathrm{O})(\mathrm{S})$ & 6.08 & $\mathrm{H} 135 \mathrm{PC}(\mathrm{OH})(\mathrm{S})$ & 6.64 & H35TOL(S) & 4.86 \\
\hline $\mathrm{H} 234 \mathrm{PC}(\mathrm{O})(\mathrm{S})$ & 2.01 & $\mathrm{H} 136 \mathrm{PC}(\mathrm{OH})(\mathrm{S})$ & 3.82 & H123TOL(S) & 3.05 \\
\hline $\mathrm{H} 235 \mathrm{PC}(\mathrm{O})(\mathrm{S})$ & 4.26 & $\mathrm{H} 145 \mathrm{PC}(\mathrm{OH})(\mathrm{S})$ & 3.90 & H124TOL(S) & 3.70 \\
\hline $\mathrm{H} 236 \mathrm{PC}(\mathrm{O})(\mathrm{S})$ & 1.86 & $\mathrm{H} 146 \mathrm{PC}(\mathrm{OH})(\mathrm{S})$ & 3.72 & H125TOL(S) & 3.87 \\
\hline $\mathrm{H} 345 \mathrm{PC}(\mathrm{O})(\mathrm{S})$ & 4.41 & $\mathrm{H} 156 \mathrm{PC}(\mathrm{OH})(\mathrm{S})$ & 3.10 & H126TOL(S) & 3.23 \\
\hline $\mathrm{H} 245 \mathrm{PC}(\mathrm{O})(\mathrm{S})$ & 4.36 & $\mathrm{H} 134 \mathrm{PC}(\mathrm{OH})(\mathrm{S})$ & 3.92 & H134TOL(S) & 3.64 \\
\hline $\mathrm{H} 2345 \mathrm{PC}(\mathrm{O})(\mathrm{S})$ & 2.76 & $\mathrm{H} 345 \mathrm{PC}(\mathrm{OH})(\mathrm{S})$ & 3.31 & H135TOL(S) & 6.47 \\
\hline $\mathrm{H} 2356 \mathrm{PC}(\mathrm{O})(\mathrm{S})$ & 2.70 & $\mathrm{H} 1234 \mathrm{PC}(\mathrm{OH})(\mathrm{S})$ & 1.92 & H236TOL(S) & 3.62 \\
\hline $\mathrm{H} 1 \mathrm{PC}(\mathrm{OH})(\mathrm{S})$ & 3.70 & $\mathrm{H} 1235 \mathrm{PC}(\mathrm{OH})(\mathrm{S})$ & 4.54 & H1234TOL(S) & 1.80 \\
\hline $\mathrm{H} 3 \mathrm{PC}(\mathrm{OH})(\mathrm{S})$ & 3.67 & $\mathrm{H} 1236 \mathrm{PC}(\mathrm{OH})(\mathrm{S})$ & 2.02 & H1235TOL(S) & 4.52 \\
\hline $\mathrm{H} 4 \mathrm{PC}(\mathrm{OH})(\mathrm{S})$ & 3.71 & $\mathrm{H} 1245 \mathrm{PC}(\mathrm{OH})(\mathrm{S})$ & 4.63 & H1236TOL(S) & 1.78 \\
\hline $\mathrm{H} 5 \mathrm{PC}(\mathrm{OH})(\mathrm{S})$ & 3.61 & $\mathrm{H} 1246 \mathrm{PC}(\mathrm{OH})(\mathrm{S})$ & 4.72 & H1245TOL(S) & 4.44 \\
\hline $\mathrm{H} 6 \mathrm{PC}(\mathrm{OH})(\mathrm{S})$ & 3.69 & $\mathrm{H} 1256 \mathrm{PC}(\mathrm{OH})(\mathrm{S})$ & 1.93 & H1246TOL(S) & 4.63 \\
\hline $\mathrm{H} 12 \mathrm{PC}(\mathrm{OH})(\mathrm{S})$ & 2.92 & $\mathrm{H} 1345 \mathrm{PC}(\mathrm{OH})(\mathrm{S})$ & 4.87 & H1345TOL(S) & 4.68 \\
\hline $\mathrm{H} 13 \mathrm{PC}(\mathrm{OH})(\mathrm{S})$ & 5.02 & $\mathrm{H} 1346 \mathrm{PC}(\mathrm{OH})(\mathrm{S})$ & 4.85 & H12346TOL(S) & 2.91 \\
\hline $\mathrm{H} 14 \mathrm{PC}(\mathrm{OH})(\mathrm{S})$ & 2.75 & $\mathrm{H} 1356 \mathrm{PC}(\mathrm{OH})(\mathrm{S})$ & 4.57 & H12356TOL(S) & 2.75 \\
\hline $\mathrm{H} 15 \mathrm{PC}(\mathrm{OH})(\mathrm{S})$ & 5.10 & $\mathrm{H} 1456 \mathrm{PC}(\mathrm{OH})(\mathrm{S})$ & 1.85 & & \\
\hline
\end{tabular}




\subsection{Lateral Interactions}

We develop a lateral interaction model for self and binary interactions as shown below:

$$
Q_{i}\left(\theta_{1}, \ldots, \theta_{n}\right)=Q_{i, 0}+2 w_{i i} \theta_{i}+\sum_{j=1, i \neq j}^{n} w_{i j} \theta_{j}
$$

where $Q_{i}$ is the binding energy of species $i, Q_{i, 0}$ is the binding energy of species $i$ in the zero coverage limit, $w_{i i}$ is the lateral interaction of species $i$ with itself, $\theta_{i}$ is the coverage of species $i, w_{i j}$ is the lateral interaction of species $i$ with species $j$. We included $\mathrm{H}-\mathrm{H}$ lateral interactions adopted from previous work on $\mathrm{Pt}(111)$ (-3 kcal/mol/monolayer). ${ }^{10}$ Here, we introduce a simple lateral interaction model shown in Figure S5. We calculated lateral interactions for various species with hydrogen by adding hydrogen to the unit cell and calculate the slopes. These species are along the minimum energy hydrogenation path. $(\mathrm{PC}(\mathrm{OH}), \mathrm{H} 2 \mathrm{PC}(\mathrm{OH}), \mathrm{H} 26 \mathrm{PC}(\mathrm{OH}), \mathrm{H} 246 \mathrm{PC}(\mathrm{OH}), \mathrm{H} 2346 \mathrm{PC}(\mathrm{OH}), \mathrm{H} 23456 \mathrm{PC}(\mathrm{OH})$, and MCHOH) We find that the lateral interactions correlate linearly with number of $\mathrm{Pt}$ atoms involved in adsorption. For microkinetic modeling, we include lateral interactions for $p$-cresol derivatives with 0,1 , or 2 hydrogenations (using the correlation in Figure S5). Our results are overall consistent with those of Sabbe et al. who found that the hydrogenation energy profile for benzene is downhill when the benzene is co-adsorbed with hydrogen as opposed to uphill at the zero-coverage limit. ${ }^{11}$

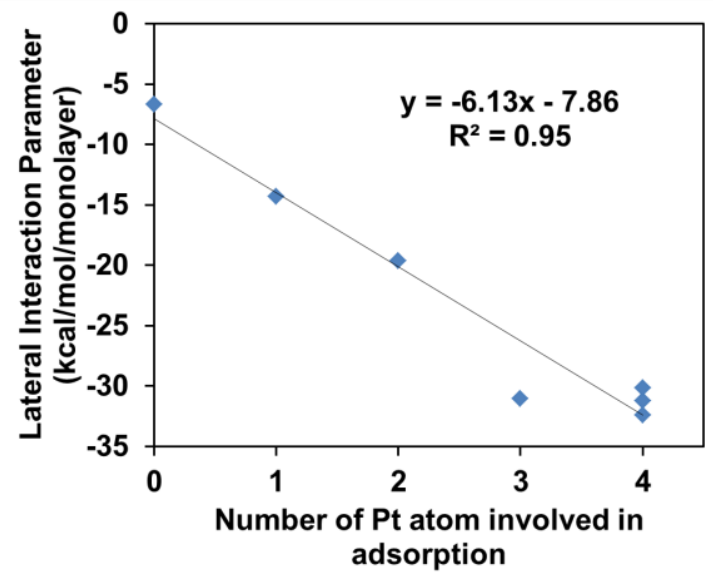

Figure S5. Lateral interaction for the effect of hydrogen coverage on the binding energy of the $p$-cresol derivatives vs. the number of $\mathrm{Pt}$ atoms involved in adsorption. 


\section{Section 4: Adsorption and Transition State Structures}

In this section, we present the adsorption and transition state structures computed via DFT. The most stable adsorption structure of $p$-cresol is bridge site, consistent with benzene. ${ }^{12}$ For the adsorption structures along the minimum energy hydrogenation path, we simply add hydrogen to the desired ring carbon position and relax the structure. We observe that the molecule remains at the same bridge site. Further addition of hydrogen at C6 creates a structure that is similar to both bridge and hollow site adsorption. For the next 4 added hydrogen atoms, the molecule relaxes to the hollow site. For toluene adsorption, we used the same adsorption configurations as $p$-cresol. For the dehydroxylated species, the adsorption configuration does not change except for the dehydroxylated $p$-cresol and H23456PC. The $\alpha$ carbon of dehydroxylated $p$-cresol moves away from the original metal atom and binds to the adjacent $\mathrm{Pt}$ atom with $\mathrm{sp}^{2}$ hybridization. The $\alpha$-carbon of dehydroxylated $\mathrm{H} 23456 \mathrm{PC}$ relaxes into the bridge site with two Pt atoms via $\sigma$ bonds.

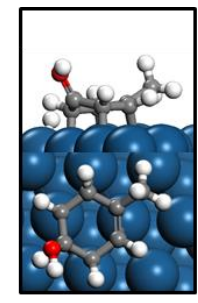

$\mathrm{H} 1 \mathrm{PC}(\mathrm{OH})$ to $\mathrm{PC}(\mathrm{OH})$

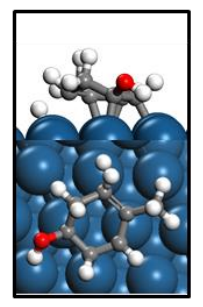

$\mathrm{H} 23 \mathrm{PC}(\mathrm{OH})$ to $\mathrm{H} 2 \mathrm{PC}(\mathrm{OH})$

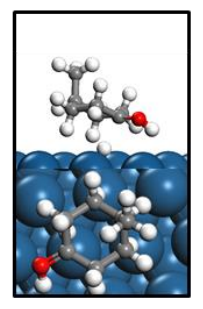

$\mathrm{MCH}(\mathrm{OH})$ to $\mathrm{H} 23456 \mathrm{PC}(\mathrm{OH})$

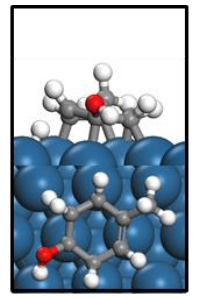

$\mathrm{H} 2 \mathrm{PC}(\mathrm{OH})$

to $\mathrm{PC}(\mathrm{OH})$
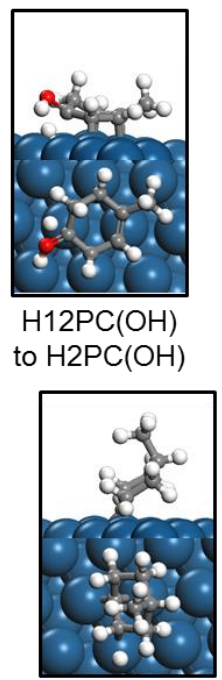

H23456PC to $\mathrm{H} 2345 \mathrm{PC}$

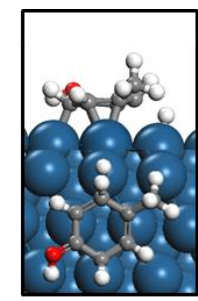

$\mathrm{H} 3 \mathrm{PC}(\mathrm{OH})$ to $\mathrm{PC}(\mathrm{OH})$

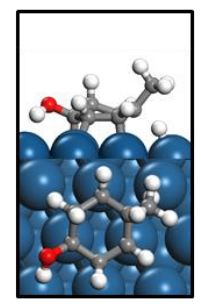

$\mathrm{H} 24 \mathrm{PC}(\mathrm{OH})$ to $\mathrm{H} 2 \mathrm{PC}(\mathrm{OH})$

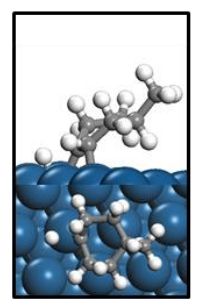

H2345TOL to $\mathrm{H} 2345 \mathrm{PC}$

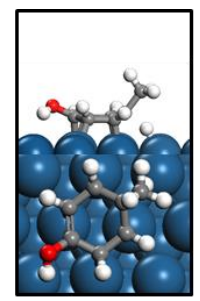

$\mathrm{H} 4 \mathrm{PC}(\mathrm{OH})$ to $\mathrm{PC}(\mathrm{OH})$

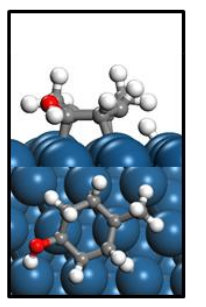

$\mathrm{H} 25 \mathrm{PC}(\mathrm{OH})$ to $\mathrm{H} 2 \mathrm{PC}(\mathrm{OH})$

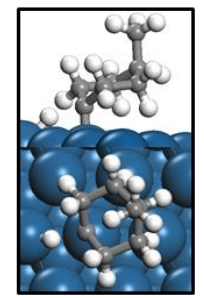

H23456TOL to $\mathrm{H} 23456 \mathrm{PC}$

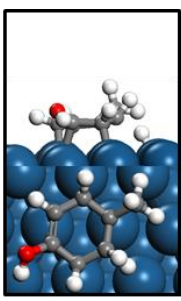

$\mathrm{H} 5 \mathrm{PC}(\mathrm{OH})$ to $\mathrm{PC}(\mathrm{OH})$

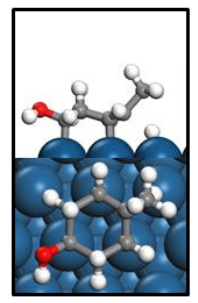

$\mathrm{H} 246 \mathrm{PC}(\mathrm{OH})$ to $\mathrm{H} 26 \mathrm{PC}(\mathrm{OH})$

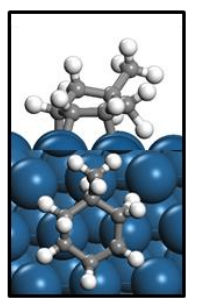

H345TOL to H34TOL

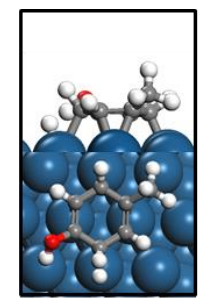

$\mathrm{H} 6 \mathrm{PC}(\mathrm{OH})$ to $\mathrm{PC}(\mathrm{OH})$

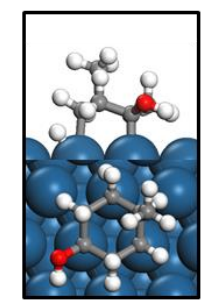

$\mathrm{H} 2346 \mathrm{PC}(\mathrm{OH})$ to $\mathrm{H} 246 \mathrm{PC}(\mathrm{OH})$

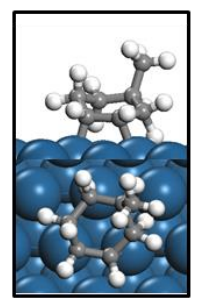

H2345TOL to $\mathrm{H} 234 \mathrm{TOL}$

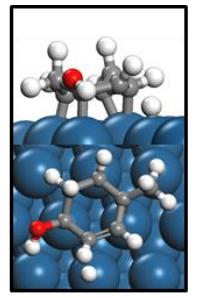

$\mathrm{H} 26 \mathrm{PC}(\mathrm{OH})$ to $\mathrm{H} 2 \mathrm{PC}(\mathrm{OH})$

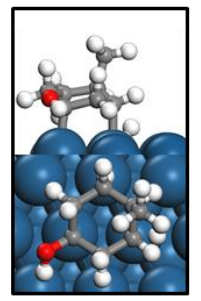

$\mathrm{H} 23456 \mathrm{PC}(\mathrm{OH})$ to $\mathrm{H} 2346 \mathrm{PC}(\mathrm{OH})$

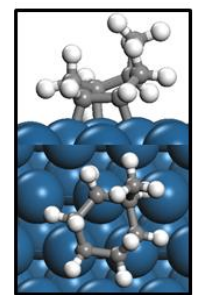

$\mathrm{H} 2345 \mathrm{TOL}$ to $\mathrm{H} 245 \mathrm{TOL}$

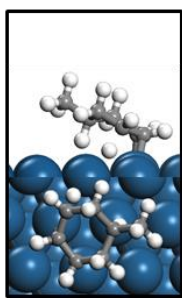

H2345TOL to H345TOL

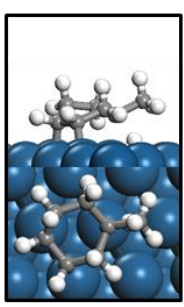

H2345TOL to H235TOL to

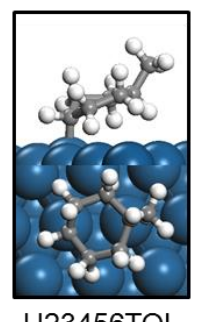

H23456TOL to $\mathrm{H} 2345 \mathrm{TOL}$
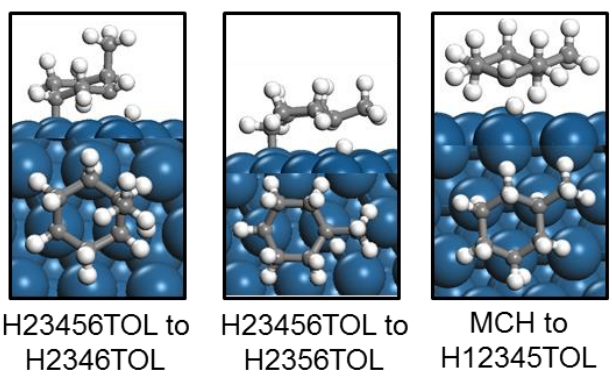

$\mathrm{MCH}$ to H12345TOL

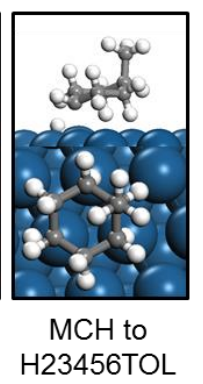

Figure S6. Transition state structures of $\mathrm{C}-\mathrm{H}$ bond scission reactions. 


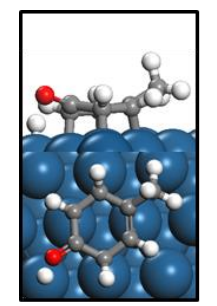

$\mathrm{PC}(\mathrm{OH})$ to $\mathrm{PC}(\mathrm{O})$

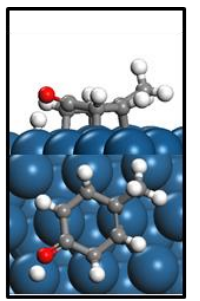

$\mathrm{PC}(\mathrm{OH})$

to $\mathrm{PC}(\mathrm{O})$ $\mathrm{C}=\mathrm{O}$ adsorbed

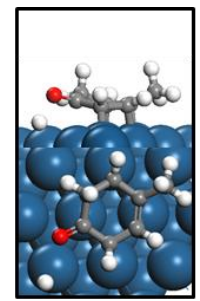

$\mathrm{H} 2 \mathrm{PC}(\mathrm{OH})$ to $\mathrm{H} 2 \mathrm{PC}(\mathrm{O})$

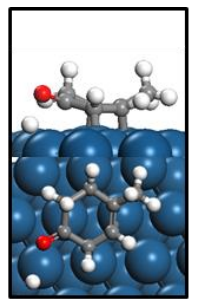

$\mathrm{H} 2 \mathrm{PC}(\mathrm{OH})$

to $\mathrm{H} 2 \mathrm{PC}(\mathrm{O})$

$\mathrm{C}=\mathrm{O}$ adsorbed

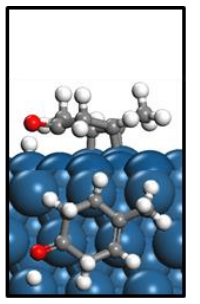

$\mathrm{H} 26 \mathrm{PC}(\mathrm{OH})$ to $\mathrm{H} 26 \mathrm{PC}(\mathrm{O})$

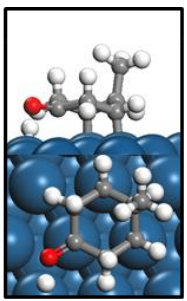

$\mathrm{H} 246 \mathrm{PC}(\mathrm{OH})$ to $\mathrm{H} 246 \mathrm{PC}(\mathrm{O})$

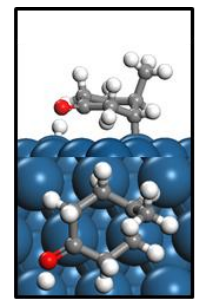

$\mathrm{H} 2346 \mathrm{PC}(\mathrm{OH})$ to $\mathrm{H} 2346 \mathrm{PC}(\mathrm{O})$

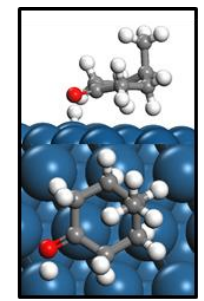

$\mathrm{H} 23456 \mathrm{PC}(\mathrm{OH})$ to $\mathrm{H} 23456 \mathrm{PC}(\mathrm{O})$

Figure S7. Transition state structures of $\mathrm{O}-\mathrm{H}$ bond scission reactions.

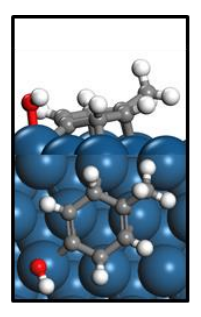

$\mathrm{PC}(\mathrm{OH})$

to $\mathrm{PC}$

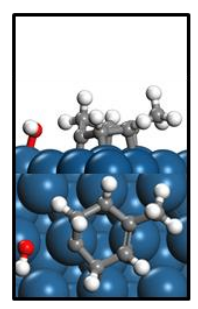

$\mathrm{H} 2 \mathrm{PC}(\mathrm{OH})-2$

to $\mathrm{H} 2 \mathrm{PC}$

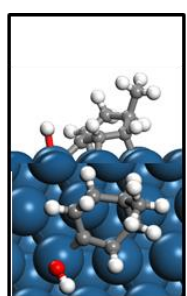

$\mathrm{H} 34 \mathrm{PC}(\mathrm{OH})-1$ to $\mathrm{H} 34 \mathrm{PC}$

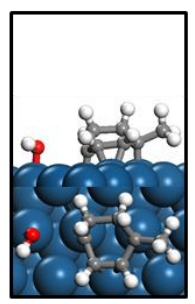

$\mathrm{H} 23 \mathrm{PC}(\mathrm{OH})$ to $\mathrm{H} 23 \mathrm{PC}$

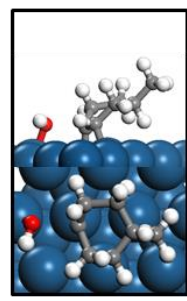

$\mathrm{H} 2345 \mathrm{PC}(\mathrm{OH})$ to $\mathrm{H} 2345 \mathrm{PC}$

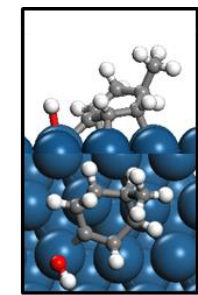

$\mathrm{H} 34 \mathrm{PC}(\mathrm{OH})-2$ to $\mathrm{H} 34 \mathrm{PC}$

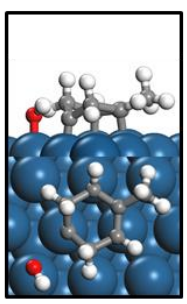

$\mathrm{H} 26 \mathrm{PC}(\mathrm{OH})$ to $\mathrm{H} 26 \mathrm{PC}$

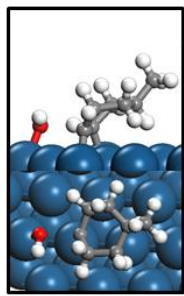

$\mathrm{H} 3456 \mathrm{PC}(\mathrm{OH})$ to $\mathrm{H} 2345 \mathrm{PC}$

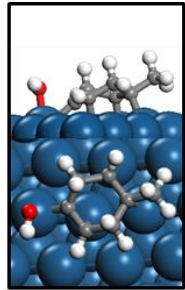

$\mathrm{H} 25 \mathrm{PC}(\mathrm{OH})$ to $\mathrm{H} 25 \mathrm{PC}$

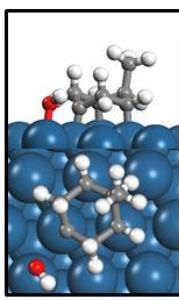

$\mathrm{H} 246 \mathrm{PC}(\mathrm{OH})$ to $\mathrm{H} 246 \mathrm{PC}$

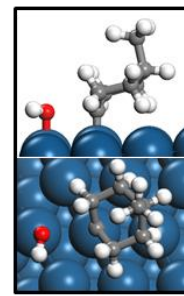

$\mathrm{H} 23456 \mathrm{PC}(\mathrm{OH})$ to $\mathrm{H} 23456 \mathrm{PC}$ ( $\mathrm{sp}^{3} \mathrm{a}$-carbon)

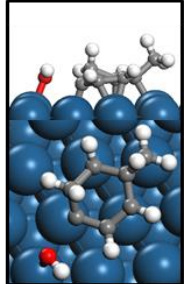

$\mathrm{H} 2 \mathrm{PC}(\mathrm{OH})-1$ to $\mathrm{H} 2 \mathrm{PC}$

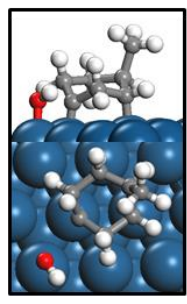

$\mathrm{H} 2346 \mathrm{PC}(\mathrm{OH})$ to $\mathrm{H} 2346 \mathrm{PC}$

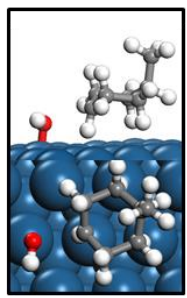

$\mathrm{MCH}(\mathrm{OH})$ to H23456TOL

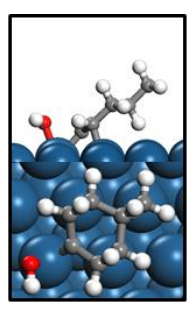

$\mathrm{H} 3456 \mathrm{PC}(\mathrm{OH})$ to $\mathrm{H} 345 \mathrm{PC}$

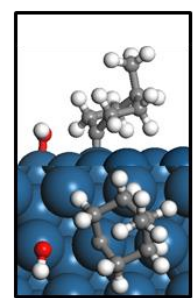

$\mathrm{H} 23456 \mathrm{PC}(\mathrm{OH})$ to $\mathrm{H} 23456 \mathrm{PC}$ (sp ${ }^{2} \alpha$-carbon)

Figure S8. Transition state structures of $\mathrm{C}-\mathrm{OH}$ bond scission reactions.

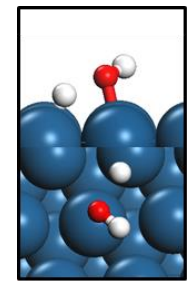

Water

Formation

Figure S9. H-OH scission transition state structure. 


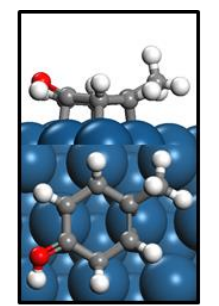

$\mathrm{PC}(\mathrm{OH})$

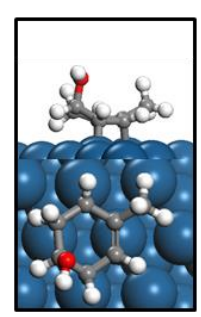

$\mathrm{H} 12 \mathrm{PC}(\mathrm{OH})$

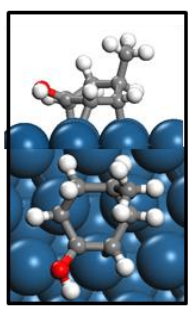

$\mathrm{H} 34 \mathrm{PC}(\mathrm{OH})-2$ (C-OH BEP)

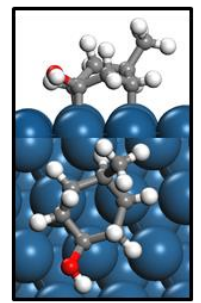

$\mathrm{H} 346 \mathrm{PC}(\mathrm{OH})$

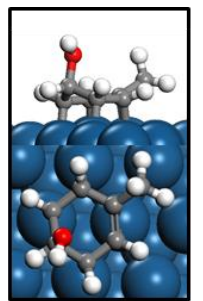

$\mathrm{H} 1 \mathrm{PC}(\mathrm{OH})$

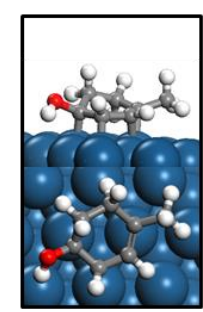

$\mathrm{H} 23 \mathrm{PC}(\mathrm{OH})-1$ (C-H BEP)

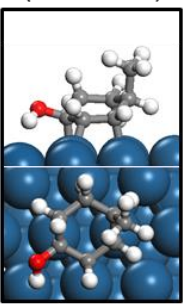

$\mathrm{H} 234 \mathrm{PC}(\mathrm{OH})$

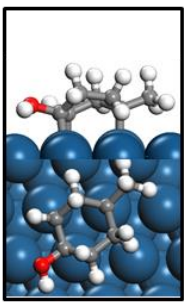

$\mathrm{H} 356 \mathrm{PC}(\mathrm{OH})$

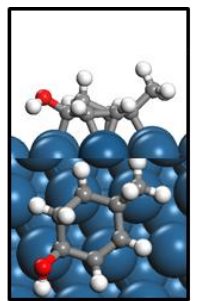

$\mathrm{H} 2 \mathrm{PC}(\mathrm{OH})-1$ (C-OH BEP)

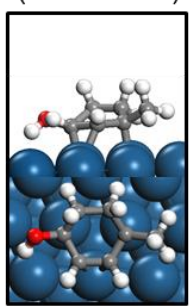

$\mathrm{H} 23 \mathrm{PC}(\mathrm{OH})-2$ (C-OH BEP)

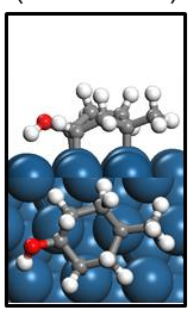

$\mathrm{H} 235 \mathrm{PC}(\mathrm{OH})$

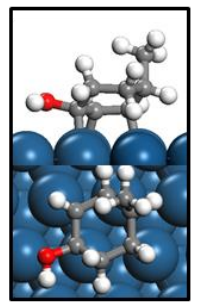

$\mathrm{H} 456 \mathrm{PC}(\mathrm{OH})$

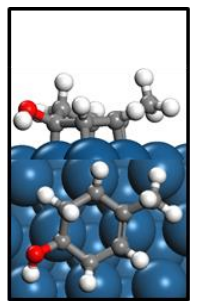

$\mathrm{H} 2 \mathrm{PC}(\mathrm{OH})-2$

(MHREP)

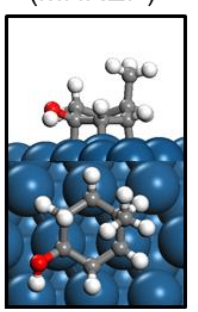

$\mathrm{H} 24 \mathrm{PC}(\mathrm{OH})$

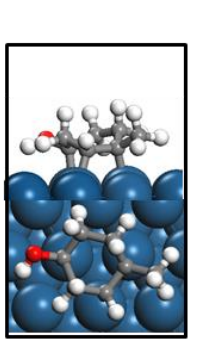

$\mathrm{H} 236 \mathrm{PC}(\mathrm{OH})$

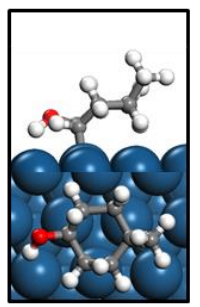

$\mathrm{H} 2345 \mathrm{PC}(\mathrm{OH})$

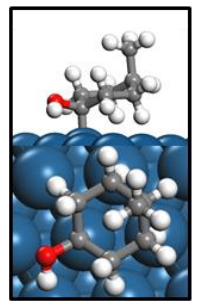

$\mathrm{H} 23456 \mathrm{PC}(\mathrm{OH})$

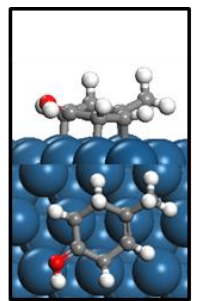

$\mathrm{H} 3 \mathrm{PC}(\mathrm{OH})$

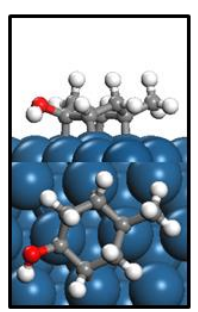

$\mathrm{H} 25 \mathrm{PC}(\mathrm{OH})-1$
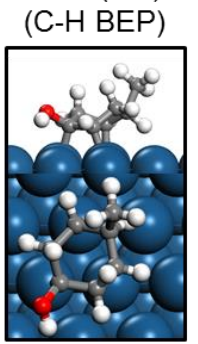

$\mathrm{H} 245 \mathrm{PC}(\mathrm{OH})$

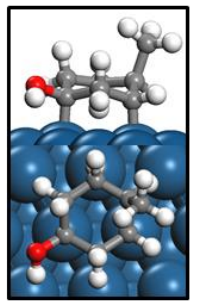

$\mathrm{H} 2346 \mathrm{PC}(\mathrm{OH})$

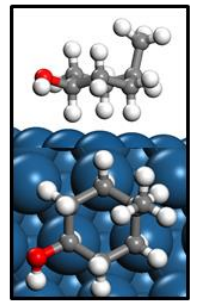

$\mathrm{MCH}(\mathrm{OH})$

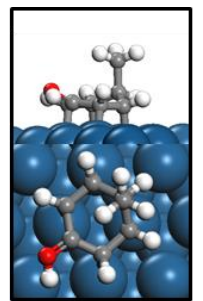

$\mathrm{H} 4 \mathrm{PC}(\mathrm{OH})$

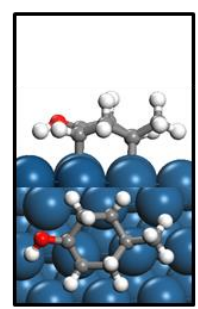

$\mathrm{H} 25 \mathrm{PC}(\mathrm{OH})-2$ (C-OH BEP)

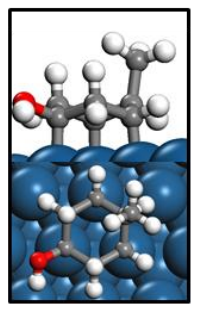

$\mathrm{H} 246 \mathrm{PC}(\mathrm{OH})$

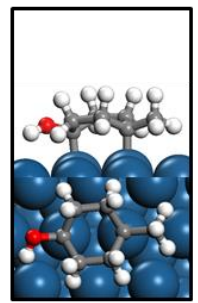

$\mathrm{H} 2356 \mathrm{PC}(\mathrm{OH})$

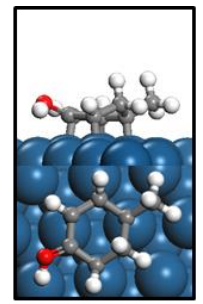

$\mathrm{H} 5 \mathrm{PC}(\mathrm{OH})$

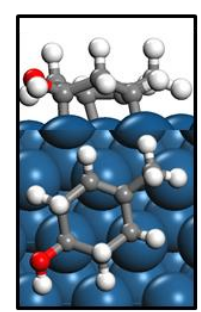

$\mathrm{H} 26 \mathrm{PC}(\mathrm{OH})$

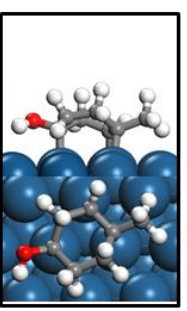

$\mathrm{H} 256 \mathrm{PC}(\mathrm{OH})$

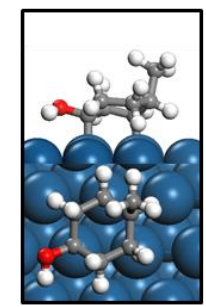

$\mathrm{H} 2456 \mathrm{PC}(\mathrm{OH}) \quad \mathrm{H} 3456 \mathrm{PC}(\mathrm{OH})$

Figure S10. Adsorption structures for the $p$-cresol derivatives. 


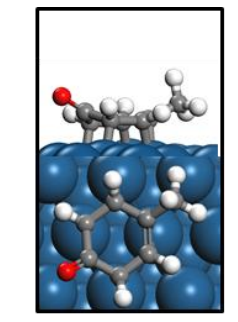

$\mathrm{PC}(\mathrm{O})$

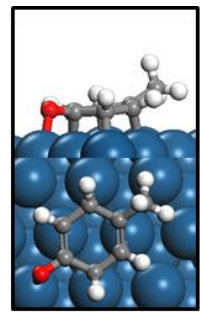

$\mathrm{PC}(\mathrm{O})$

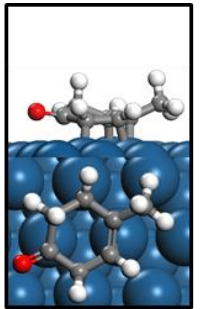

$\mathrm{H} 2 \mathrm{PC}(\mathrm{O})$

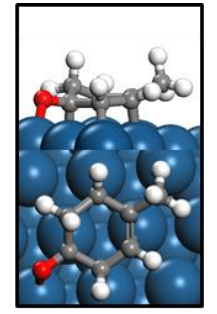

$\mathrm{H} 2 \mathrm{PC}(\mathrm{O})$

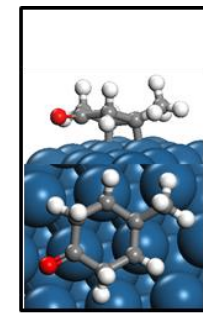

$\mathrm{H} 26 \mathrm{PC}(\mathrm{O})$

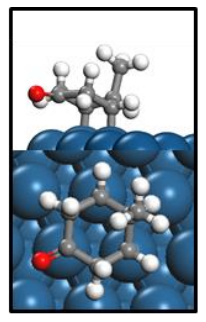

$\mathrm{H} 246 \mathrm{PC}(\mathrm{O})$

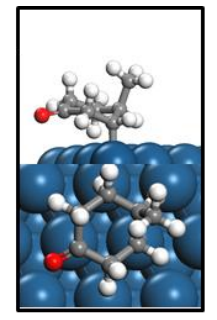

$\mathrm{H} 2346 \mathrm{PC}(\mathrm{O})$

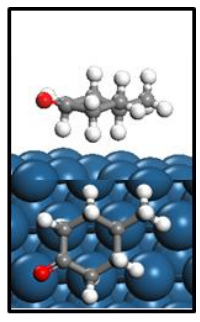

$\mathrm{MCH}(\mathrm{O})$

Figure S11. Adsorption structures of the $p$-cresol derivatives with $\mathrm{H}$ deficient oxygen.

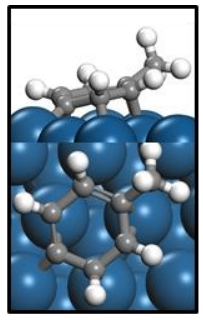

PC

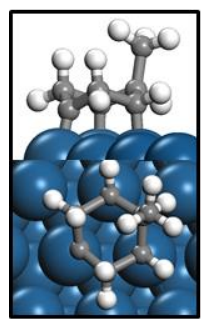

H246PC

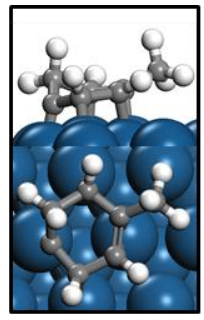

$\mathrm{H} 2 \mathrm{PC}$

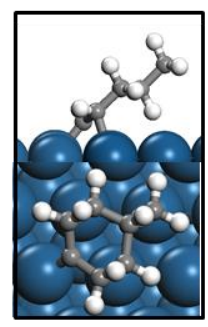

H345PC

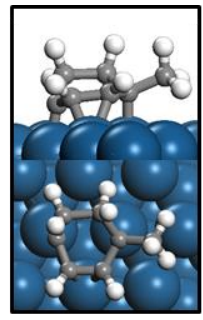

$\mathrm{H} 23 \mathrm{PC}$

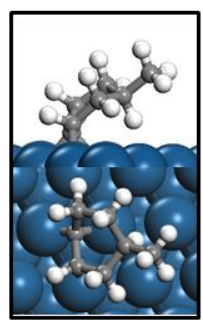

H2345PC

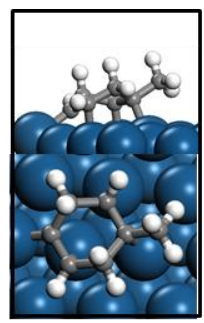

H25PC

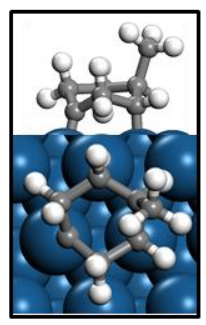

H2346PC

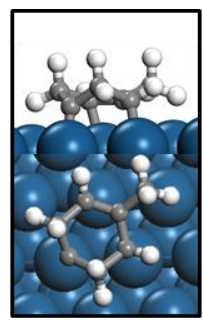

$\mathrm{H} 26 \mathrm{PC}$

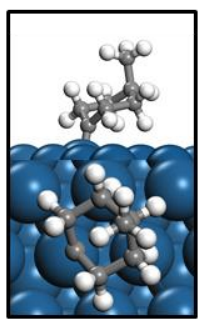

$\mathrm{H} 23456 \mathrm{PC}$ (sp ${ }^{2} \alpha$-carbon)

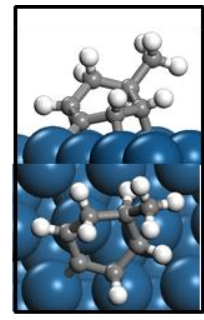

H34PC

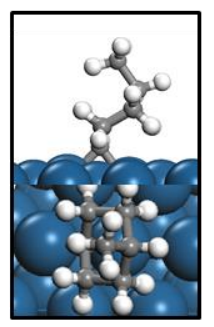

H23456PC

(sp ${ }^{3} \mathrm{\alpha}$-carbon)

Figure S12. Adsorption structures of the $p$-cresol derivatives with radical $\alpha$-carbon.

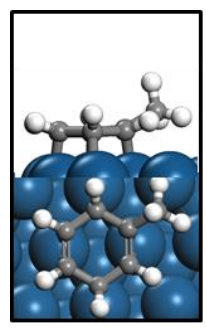

TOL

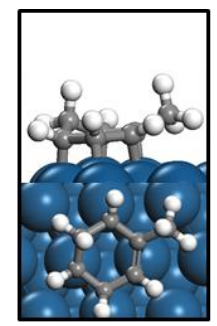

H2TOL

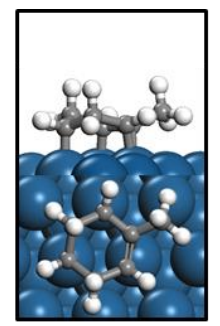

H26TOL

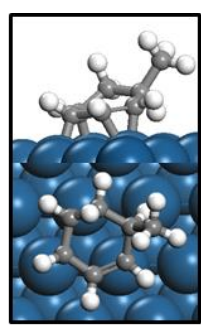

H34TOL

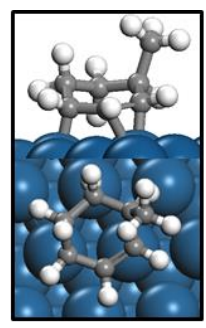

H234TOL

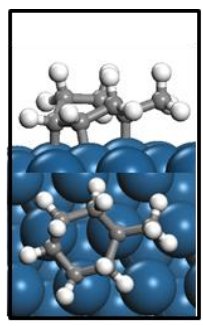

H235TOL

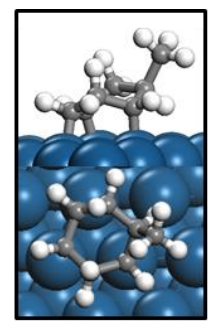

H245TOL

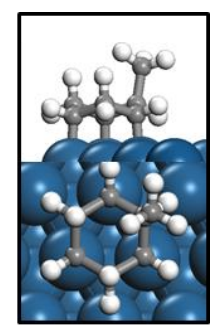

H246TOL

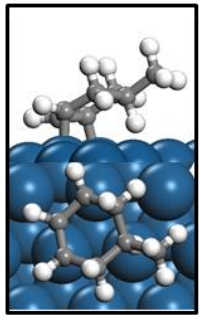

H345TOL

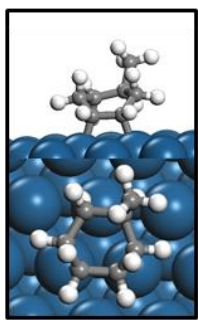

H2345TOL

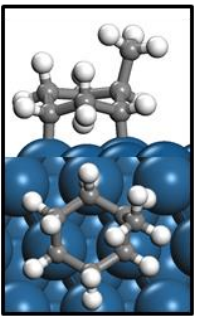

H2346TOL

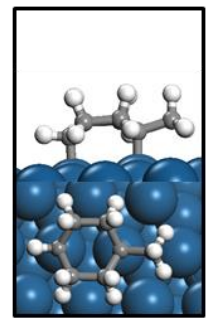

H2356TOL

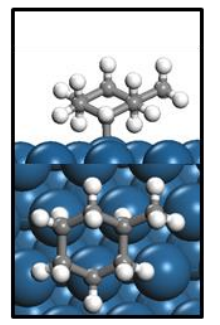

H12345TOL

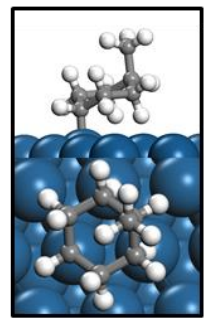

H23456TOL

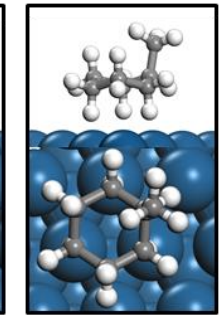

$\mathrm{MCH}$

Figure S13. Adsorption structures for the toluene derivatives. 


\section{Section 5: MKM Parameters Adjustments}

Table S7. List of parameter adjustments for the MKM model.

\section{Adjusted parameter}

$\Delta H_{f}$ of methyl cyclohexanone

$\Delta H_{f}$ of methyl cyclohexanol

Pre-exp. of methyl cyclohexanol desorption

Pre-exp. of methyl cyclohexanone desorption

Pre-exp. of C-OH scission reactions

Pre-exp. of C-H scission reactions for toluene

derivatives with 1or 2 hydrogenated ring

carbons

\section{Amount of adjustments}

$-0.14 \mathrm{eV}$

$-0.12 \mathrm{eV}$

40 fold (equivalent to $0.18 \mathrm{eV}$ decrease in barrier)

5 fold (equivalent to $0.08 \mathrm{eV}$ decrease in barrier)

0.5 fold (equivalent to $0.03 \mathrm{eV}$ increase in barrier)

10 fold (equivalent to $0.11 \mathrm{eV}$ decrease in barrier)

\section{Section 6: Reaction Energies}

(a)

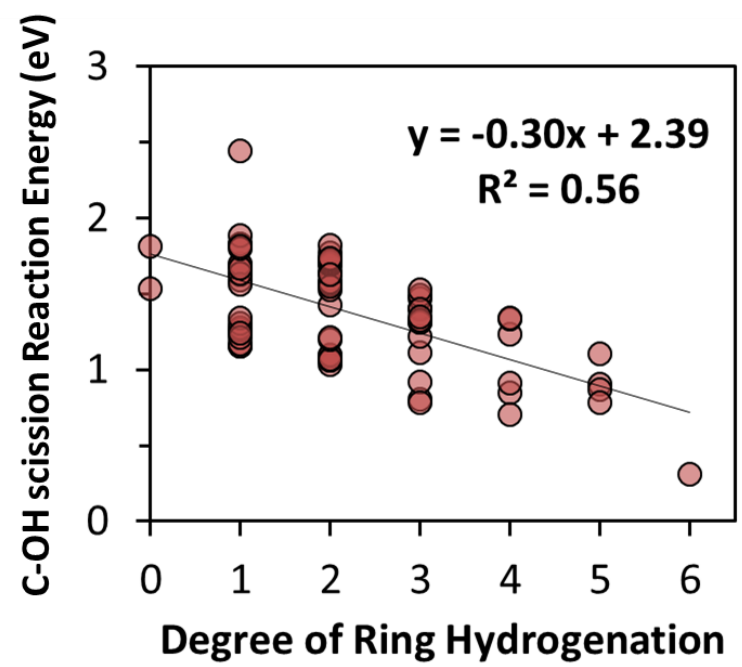

(b)

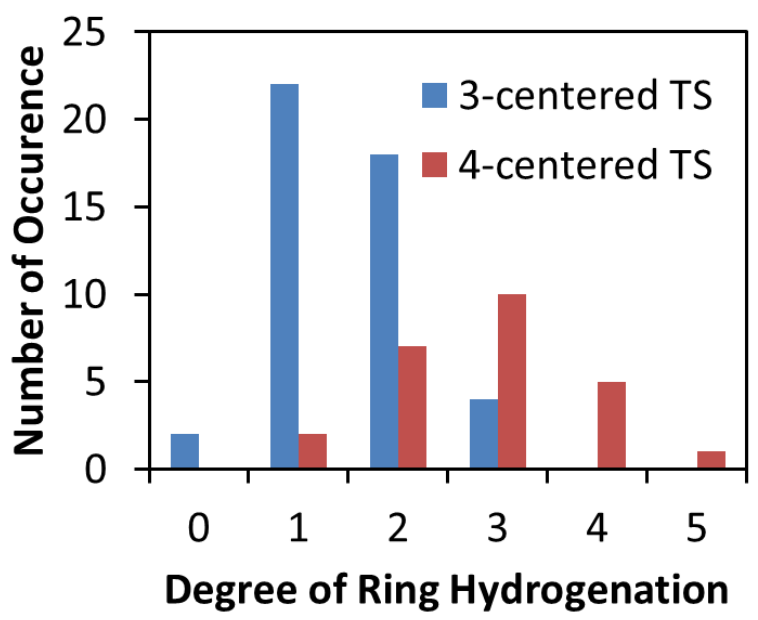

Figure S14. (a) C-OH scission reaction energy vs. degree of ring hydrogenation for the alcohols in the entire reaction network. (b) Number of $\mathrm{C}-\mathrm{OH}$ scission reactions encountered vs. degree of ring hydrogenation. The combination of these two factors leads to efficient $\mathrm{C}-\mathrm{OH}$ scission upon sufficient ring hydrogenation. 


\section{Section 7: MKM Results}

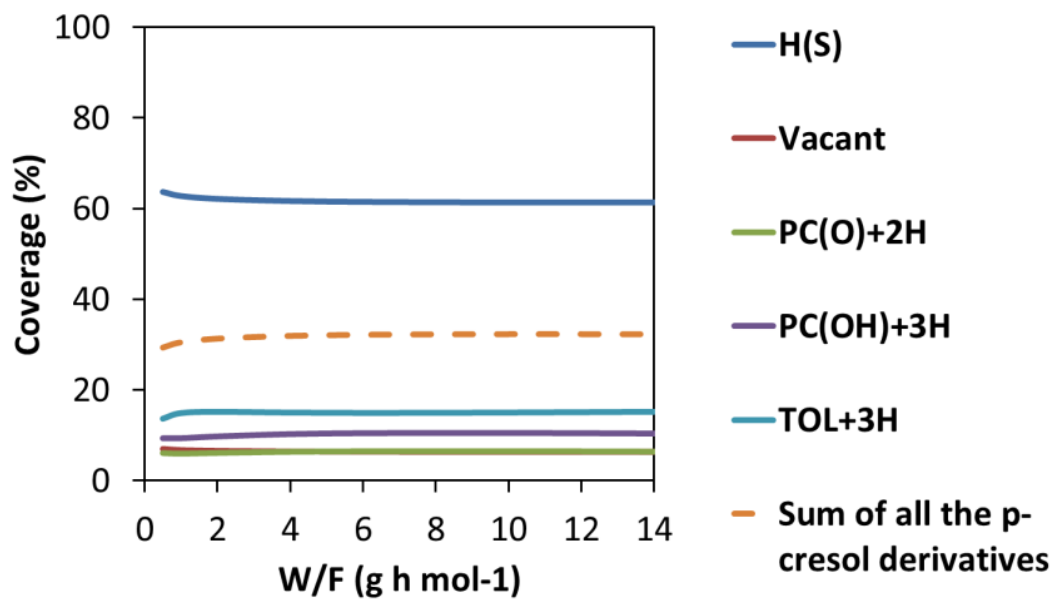

Figure S15. Surface coverages of $p$-cresol simulation for Nie et al. experimental data ${ }^{4}$ shown in Figure 5 in the main text. Hydrogen is the most dominant species on the surface, followed by toluene derivatives with 3 hydrogenated ring carbons $(\mathrm{TOL}+3 \mathrm{H}), p$-cresol with 3 hydrogenated ring carbons $(\mathrm{PC}(\mathrm{OH})+3 \mathrm{H})$, and $p$-cresol with $\mathrm{H}$ deficient oxygen and 2 hydrogenated ring carbons $(\mathrm{PC}(\mathrm{O})+2 \mathrm{H})$.

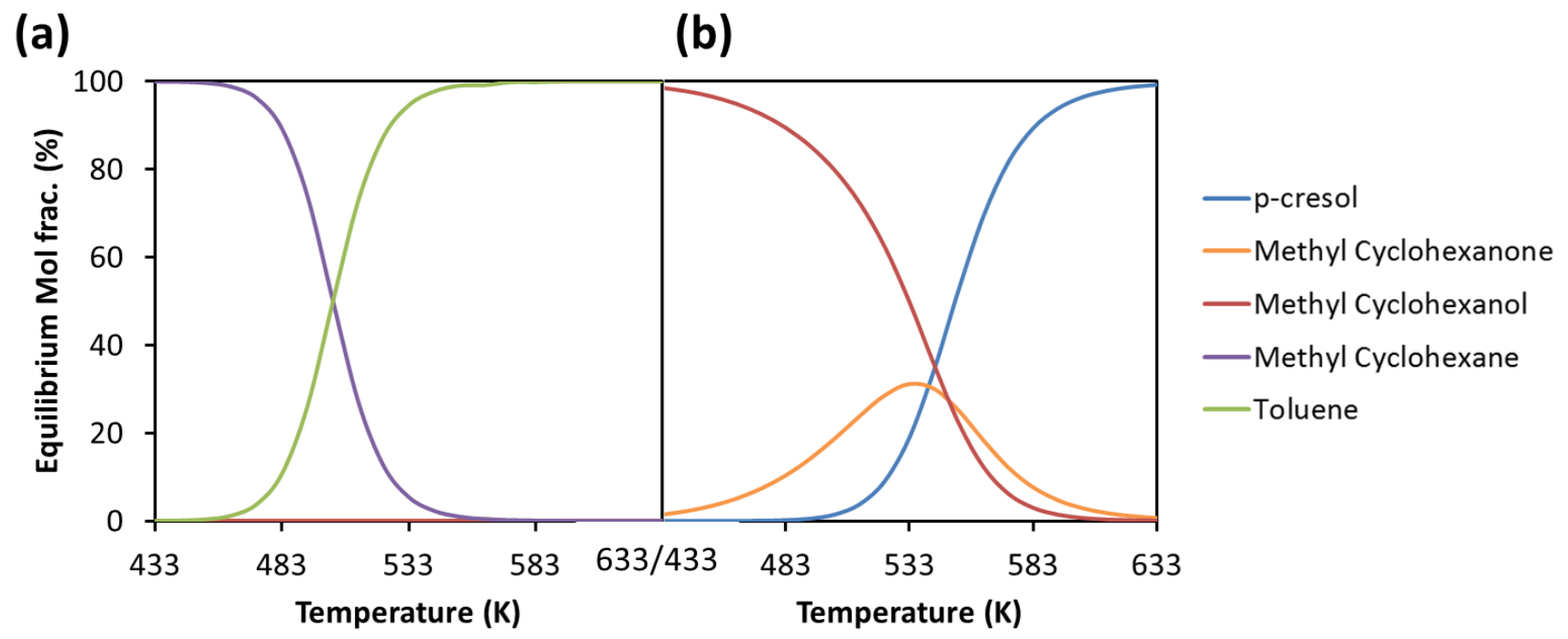

Figure S16. Molar equilibrium composition with (a) and without (b) hydrocarbon products accounted for. Panel (b) is reminiscent of near the entrance region of the reactor. Conditions are $1.25 \times 10^{-2} \mathrm{~atm} p$ cresol, $1.0 \mathrm{~atm} \mathrm{H}_{2}$. 
REFERENCES

(1) Rangarajan, S.; Bhan, A.; Daoutidis, P. Comput. Chem. Eng. 2012, 46, 141-152.

(2) Rangarajan, S.; Bhan, A.; Daoutidis, P. Comput. Chem. Eng. 2012, 45, 114-123.

(3) Foster, A.; Do, P. M.; Lobo, R. Top. Catal. 2012, 55, 118-128.

(4) Nie, L.; Resasco, D. E. J. Catal. 2014, 317, 22-29.

(5) Sutton, J. E.; Vlachos, D. G. ACS Catal. 2012, 2, 1624-1634.

(6) Gao, W.; Zheng, W. T.; Jiang, Q. J. Chem. Phys. 2008, 129, 164705.

(7) Kandoi, S.; Greeley, J.; Sanchez-Castillo, M. A.; Evans, S. T.; Gokhale, A. A.; Dumesic, J. A.; Mavrikakis, M. Top. Catal. 2006, 37, 17-28.

(8) Mhadeshwar, A. B.; Vlachos, D. G. J. Catal. 2005, 234, 48-63.

(9) Salciccioli, M.; Chen, Y.; Vlachos, D. G. Ind. Eng. Chem. Res. 2011, 50, 28-40.

(10) Sutton, J. E.; Panagiotopoulou, P.; Verykios, X. E.; Vlachos, D. G. J. Phys. Chem. C 2013, 117, 4691-4706.

(11) Sabbe, M. K.; Canduela-Rodriguez, G.; Reyniers, M.-F.; Marin, G. B. J. Catal. 2015, 330, 406422.

(12) Morin, C.; Simon, D.; Sautet, P. Surf. Sci. 2006, 600, 1339-1350.

19 